\title{
Fermionic Bell violation in the presence of background electromagnetic fields in the cosmological de Sitter spacetime
}

\author{
Md Sabir Ali@, ${ }^{*}$ Sourav Bhattacharya $\odot,{ }^{\dagger}$ Shankhadeep Chakrabortty, ${ }^{\ddagger}$ and Shagun Kaushal ${ }^{\S}$ \\ Department of Physics, Indian Institute of Technology Ropar, Rupnagar, Punjab 140 001, India
}

(Received 3 March 2021; accepted 12 November 2021; published 14 December 2021)

\begin{abstract}
The violation of the Bell inequality for Dirac fermions is investigated in the cosmological de Sitter spacetime, in the presence of background electromagnetic fields of constant strengths. The orthonormal Dirac mode functions are obtained and the relevant in-out squeezed state expansion in terms of the Bogoliubov coefficients are found. We focus on two scenarios here: strong electric field and heavy mass limits (with respect to the Hubble constant). Using the squeezed state expansion, we then demonstrate the Bell violations for the vacuum and some maximally entangled initial states. Even though a background magnetic field alone cannot create particles, in the presence of background electric field and or spacetime curvature, it can affect the particle creation rate. Thus, our chief aim here is to investigate the role of the background magnetic field strength in the Bell violation. Qualitative differences in this regard for different maximally entangled initial states are shown. Further extension of these results to the so-called $\alpha$ vacua are also discussed.
\end{abstract}

DOI: 10.1103/PhysRevD.104.125012

\section{INTRODUCTION}

One of the most outstanding features of quantum mechanics is certainly the entanglement, associated with the nonlocal properties of the quantum mechanical measurement procedure [1-9]. After experimental confirmation, this has been placed on firm physical grounds [10,11]. We refer our readers to, e.g., [12-17] and references therein for extensive reviews and pedagogical discussions on quantum entanglement and its various measures.

A very important and useful measure of quantum entanglement is the violation of the Bell inequality $[2,3]$ (see also [16] for an excellent pedagogical discussion), which has been confirmed experimentally $[10,11]$. Such violation clearly rules out the so-called classical hidden variable theories and establishes the probabilistic and (for entangled states) the nonlocal characteristics associated with the quantum measurement procedure, e.g., $[6,8,9]$ (also references therein). The Bell inequality was originally designed for bipartite pure states, which was later extended to multipartite systems, altogether known as the

\footnotetext{
*sabir.ali@iitrpr.ac.in

†sbhatta@iitrpr.ac.in

s.chakrabortty@iitrpr.ac.in

§2018phz0006@iitrpr.ac.in
}

Published by the American Physical Society under the terms of the Creative Commons Attribution 4.0 International license. Further distribution of this work must maintain attribution to the author(s) and the published article's title, journal citation, and DOI. Funded by SCOAP .
Bell-Mermin-Klyshko inequalities (or the Clauser-HorneShimony-Holt inequality) [3,12-14].

There are a couple of distinct relativistic sectors where entanglement properties of quantum fields emerge very naturally, due to the creation of entangled particle pairs. The first is the maximally extended nonextremal black hole spacetimes, or the Rindler spacetime, where the entanglement of quantum fields between two causally disconnected spacetime wedges have been investigated, e.g., [18-22]. The second is the cosmological backgrounds where the vacuum in the asymptotic future (the out vacuum) is related to that of the asymptotic past (the in vacuum) via squeezed state expansion, due to pair creation. We refer our reader to, e.g., [23-32] and references therein for discussions on various measures of bosonic and fermionic fields in different coordinatization of the de Sitter spacetime. Even in the flat spacetime particle pair creation is possible in the presence of a "sufficiently" strong background electric field, viz. the Schwinger pair creation, e.g., [33]. Various aspects of entanglement properties between created particle-antiparticle pairs in the Schwinger mechanism, including the effect of a background magnetic field, can be seen in [34-40]. We also refer our readers to, e.g., [41,42] for interesting aspects of entanglement in the flat space quantum field theory and to $[43,44]$ for holographic aspects of entanglement.

The study of entanglement in the context of the early inflationary era can give us insight about the state of a quantum field in the early Universe. Such investigations should not be regarded as mere academic interests, as attempts have been made to predict their possible observational signatures as well. Specifically, entanglement 
generated in the early Universe can affect the cosmological correlation functions or the cosmic microwave background (CMB). For example, the fermionic entanglement may lead to the breaking of scale invariance of the inflationary power spectra [45]. It was argued in [46] by studying the violation of the Bell inequality by the photons coming from certain high redshift quasars that they are entangled, indicating the existence of entangled quantum states in the early Universe. We also refer our readers to [47] and references therein for discussion on signature of Bell violation in the CMB and its observational constraints pertaining the Bell operators and some course graining parameter.

In the cosmological spacetimes, particle pair creation occurs due to the background spacetime curvature, e.g., [33]. However if background electromagnetic fields are also present there, the particle creation can further be affected. A particularly interesting scenario is the early inflationary spacetime endowed with primordial electromagnetic fields. Computations on the Schwinger effect for both bosonic and fermionic fields in the de Sitter spacetime and its possible connection to the observed magnetic field in the intergalactic spaces (i.e., the so-called Galactic dynamo problem [48]) can be seen in, e.g., [49-54].

In this paper, we wish to compute the Bell violation for fermions in the cosmological de Sitter spacetime, in the presence of constant background electromagnetic fields. Previous studies on cosmological Bell violation can be seen in, e.g., $[24,25,30]$. Note that a magnetic field alone cannot create vacuum instability [33] and can be intuitively understood as follows. Let us imagine a particleantiparticle pair is created due to the application of a magnetic field. They must move in opposite directions to get separated. However, the magnetic Lorentz force $e \vec{v} \times \vec{B}$ acts in the same direction for both particle and antiparticle. Thus by applying a magnetic field alone, no matter how strong it is, we cannot create pairs. However, one may expect that in the presence of background spacetime curvature and/or electric field, it can affect the pair creation rate. The entanglement will also certainly vary if the pair creation rate is altered.

In a flat spacetime, pair creation only due to a background electric field is expected to cease upon the application of a magnetic field of sufficiently high strength, due to the aforementioned oppositely directed Lorentz force created by them. Accordingly, the degradation of correlation or information between entangled states due to particle creation would also cease, as has been shown recently in [40]. Let us now consider, in addition, the spacetime curvature that would also create particle pairs. Will the magnetic field be able to stop the particle creation due to the gravitational field? The intuitive answer is no, as follows. In a pure gravitational background, a created particle pair will follow geodesics and become observables in a spacetime like the de Sitter spacetime due to the geodesic deviation [55]. Such deviations happen even for initially parallel trajectories. Thus as the particle-antiparticle pair created in the presence of geometric curvature propagates, they are expected to get separated irrespective of the presence of Lorentz force imparted by the background magnetic field, even though that force is acting in the same direction for both of them. This also indicates that, in the absence of an electric field, the magnetic field perhaps cannot affect the particle creation due to the gravitational field at all. We shall check these intuitive guesses explicitly in the next section. Our goal here is to study the effect of the background magnetic field strength on the Bell violation.

Apart from this, a physical motivation behind this study comes from the possible connection between the primordial electromagnetic fields and the aforementioned Galactic dynamo problem, e.g., [50]. We wish to consider fermions instead of a complex scalar, as the former are more realistic. Let us speculate about some possible observational consequences of the model we study. For example, one can compute the power spectra by tracing out the fermionic degrees of freedom (interacting with the inflaton or gravitational excitations) and check the breaking of scale invariance as in [45]. Likewise, if we also consider the quantum part of the electromagnetic sector, it should carry information about the entangled fermionic states once we trace out the fermionic degrees of freedom, originating from the photon-fermion interaction. Thus one can expect that the photons coming from the distant past undergoing the Bell test, as in [46], will carry information about such entangled fermionic states. Since these states are defined in the presence of the primordial background electromagnetic fields, the Bell test might also carry information about those background fields. This can possibly be used to constrain the corresponding field strengths and test the proposition of [50]. With this motivation, and as a problem to begin with, we shall simply compute below the fermionic Bell violation in the cosmological de Sitter spacetime in the presence of background electromagnetic fields, as a viable measure of quantum entanglement.

The rest of the paper is organized as follows. In Sec. II and the Appendix, we compute the orthonormal in and out Dirac modes in the cosmological de Sitter spacetime in the presence of constant background electric and magnetic fields. The Bogoliubov coefficients and the squeezed state relationship between the in and out vacua are also found. Using this, we compute the vacuum entanglement entropy in Sec. III. The Bell inequality violations for the vacuum and also two maximally entangled initial states are computed in Sec. IV. All these results are further extended in Sec. V to the so-called one parameter fermionic $\alpha$ vacua. Finally we conclude in Sec. VI. We shall assume either the field is heavily massive or the electric field strength is very high (with respect to the Hubble constant).

We shall work with the mostly positive signature of the metric in $(3+1)$ dimensions and will set $c=1=\hbar$ throughout. 


\section{THE IN AND OUT DIRAC MODES}

For our purpose, we first need to solve the Dirac equation in the cosmological de Sitter spacetime in the presence of constant background electromagnetic fields. The following will be an extension of the solutions found earlier in the same spacetime but in the absence of any magnetic field $[51,53]$.

The Dirac equation in a general curved spacetime reads,

$$
\left(i \gamma^{\mu} D_{\mu}-m\right) \psi(x)=0,
$$

where the gauge cum spin covariant derivative reads,

$$
D_{\mu} \equiv \partial_{\mu}+i e A_{\mu}+\Gamma_{\mu}
$$

The spin connection is given by

$$
\Gamma_{\mu}=-\frac{1}{8} e_{a}^{\mu}\left(\partial_{\mu} e_{b \nu}-\Gamma_{\mu \nu}^{\lambda} e_{b \lambda}\right)\left[\gamma^{a}, \gamma^{b}\right]
$$

where the latin indices represent the local inertial frame and $e_{a}^{\mu}$ are the tetrads.

The de Sitter spacetime metric in $(3+1)$ dimensions reads

$$
d s^{2}=\frac{1}{H^{2} \eta^{2}}\left(-d \eta^{2}+d x^{2}+d y^{2}+d z^{2}\right),
$$

where $H$ is the Hubble constant and the conformal time $\eta$ varies from $-\infty<\eta<0^{-}$. Choosing now $e_{\mu}^{a}=a(\eta) \delta_{\mu}^{a}$, we have from (2),

$$
\Gamma_{\mu}=\frac{1}{2} \gamma^{\mu} \gamma^{0} a^{\prime}(\eta) a(\eta) \delta_{\mu}^{i}, \quad i=1,2,3,
$$

where the prime denotes differentiation once with respect to $\eta$.

Defining a new variable in terms of the scale factor $a(\eta)=-1 / H \eta$ as

$$
\xi=a^{\frac{3}{2}} \psi
$$

and using (4), the Dirac equation (1) becomes,

$$
\left(i e_{a}^{\mu} \gamma^{a} \partial_{\mu}-e A_{\mu} e_{a}^{\mu} \gamma^{a}-m\right) \xi(\eta, \vec{x})=0 .
$$

Substituting next

$$
\xi(\eta, \vec{x})=\left(i e_{a}^{\mu} \gamma^{a} \partial_{\mu}-e A_{\mu} e_{a}^{\mu} \gamma^{a}+m\right) \zeta(\eta, \vec{x})
$$

into (6), we obtain the squared Dirac equation

$$
\begin{aligned}
& {\left[\left(\partial_{\mu}+i e A_{\mu}\right)^{2}-m^{2} a^{2}\right.} \\
& \left.\quad+i\left(m a^{\prime} a e_{0}^{0} \gamma^{0}-\frac{e}{2} a^{2} e_{a}^{\mu} \gamma^{a} e_{a}^{\nu} \gamma^{b} F_{\mu \nu}\right)\right] \zeta(\eta, \vec{x})=0 .
\end{aligned}
$$

We choose the gauge to obtain constant electric and magnetic fields in the $z$ direction as

$$
A_{\mu}=B y \delta_{\mu}^{x}-\frac{E}{H}(a-1) \delta_{\mu}^{z},
$$

where $E$ and $B$ are constants. Making now the ansatz

$$
\zeta(\eta, \vec{x})=e^{-i e z E / H} e^{i \vec{k}_{\curlyvee} \cdot \vec{x}_{\zeta}}(\eta, y) \omega_{s}
$$

in (8), where $\vec{k}_{y}=\left(k_{x}, 0, k_{z}\right)$, we have

$$
\left[\left(\partial_{y}^{2}-\left(k_{x}+e B y\right)^{2}\right)-\partial_{0}^{2}-k_{z}^{2}+2 H L a k_{z}-H^{2} L^{2} a^{2}-m^{2} a^{2}+i H a^{2}\left(M \gamma^{0}+L \gamma^{0} \gamma^{3}+\frac{e B}{H a^{2}} \gamma^{1} \gamma^{2}\right)\right] \zeta_{s}(\eta, y) \omega_{s}=0
$$

where

$$
M=\frac{m}{H} \quad \text { and } \quad L=\frac{e E}{H^{2}}
$$

are dimensionless mass and electric field strengths. Note also in (10) that the matrices $\left(M \gamma^{0}+L \gamma^{0} \gamma^{3}\right)$ and $\gamma^{1} \gamma^{2}$ commute and hence we may treat $\omega_{s}$ to be their simultaneous eigenvectors. Thus (10) becomes

$$
\left[\left(\partial_{y}^{2}-\left(k_{x}+e B y\right)^{2}\right)+\left(-\partial_{0}^{2}-\omega_{k}^{2}+i \lambda_{s} \sigma(\eta)+i e \beta_{s} B\right)\right] \zeta_{s}(\eta, y)=0
$$

where $\lambda_{s}= \pm 1, \beta_{s}= \pm i$ and we have abbreviated

$$
\omega_{k}^{2}=k_{z}^{2}-2 H L a k_{z}+H^{2} a^{2}\left(L^{2}+M^{2}\right), \quad \sigma(\eta)=a^{2} H^{2} \sqrt{L^{2}+M^{2}} .
$$

The explicit expressions for the four orthonormal eigenvectors $\omega_{s}$ are given in the Appendix. Substituting now for the variable separation, $\zeta_{s}(\eta, y)=\varsigma_{s}(\eta) h_{s}(y)$ into (11), we obtain the decoupled equations 


$$
\left(\partial_{\eta}^{2}+\omega_{k}^{2}-i \lambda_{s} \sigma(\eta)+S_{s}\right) \varsigma_{s}(\eta)=0 \quad \text { and } \quad\left(\partial_{y}^{2}-\left(k_{x}+e B y\right)^{2}+S_{s}+i \beta_{s} e B\right) h_{s}(y)=0
$$

where $S_{s}$ is the separation constant. Clearly, we can have four sets of such pair of equations corresponding to the different choices of $\lambda_{s}= \pm 1$ and $\beta_{s}= \pm i$. For example, for $\lambda_{s}=1, \beta_{s}=-i$ and $\lambda_{s}=1, \beta_{s}=i$, we, respectively, have

$$
\begin{aligned}
& \left(\partial_{\eta}^{2}+\omega_{k}^{2}-i \sigma(\eta)+S_{1}\right) \varsigma_{1}(\eta)=0 \quad \text { and } \quad\left(\partial_{y}^{2}-\left(k_{x}+e B y\right)^{2}+S_{1}+e B\right) h_{1}(y)=0, \\
& \left(\partial_{\eta}^{2}+\omega_{k}^{2}-i \sigma(\eta)+S_{2}\right) \varsigma_{2}(\eta)=0 \quad \text { and } \quad\left(\partial_{y}^{2}-\left(k_{x}+e B y\right)^{2}+S_{2}-e B\right) h_{2}(y)=0 .
\end{aligned}
$$

Let us first focus on the spatial equations. In terms of the variable

$$
\bar{y}=\left(\sqrt{e B} y+\frac{k_{x}}{\sqrt{e B}}\right),
$$

it is easy to see that the spatial differential equations of (14) reduce to the Hermite differential equation, with the separation constants,

$$
S_{1}=2 n e B \quad \text { and } \quad S_{2}=2(n+1) e B
$$

where $n=0,1,2 \ldots$ denote the Landau levels. Thus we have the normalized solutions

$$
h_{1}(y)=h_{2}(y)=\left(\frac{\sqrt{e B}}{2^{n+1} \sqrt{\pi}(n+1) !}\right)^{1 / 2} e^{-\bar{y}^{2} / 2} \mathcal{H}_{n}(\bar{y})=h_{n}(\bar{y})(\text { say }),
$$

where $\mathcal{H}_{n}(\bar{y})$ are the Hermite polynomials of order $n$.

For the two temporal equations in (14), we introduce the variables

$$
z_{1}=-\frac{2 i \sqrt{k_{z}^{2}+S_{1}}}{a H} \quad \text { and } \quad z_{2}=-\frac{2 i \sqrt{k_{z}^{2}+S_{2}}}{a H}
$$

so that they, respectively, become

$$
\left(\partial_{z_{1}}^{2}-\frac{1}{4}+\frac{\kappa_{1}}{z_{1}}+\frac{\left(1 / 4-\mu^{2}\right)}{z_{1}^{2}}\right) \varsigma_{1}\left(z_{1}\right)=0 \quad \text { and } \quad\left(\partial_{z_{2}}^{2}-\frac{1}{4}+\frac{\kappa_{2}}{z_{2}}+\frac{\left(1 / 4-\mu^{2}\right)}{z_{2}^{2}}\right) \varsigma_{2}\left(z_{2}\right)=0,
$$

where we have abbreviated

$$
\kappa_{1}=-\frac{i k_{z} L}{\sqrt{k_{z}^{2}+S_{1}}}, \quad \kappa_{2}=-\frac{i k_{z} L}{\sqrt{k_{z}^{2}+S_{2}}}, \quad \mu=\left(\frac{1}{2}+i \sqrt{M^{2}+L^{2}}\right)
$$

Note that $\kappa_{1,2}$ depend upon the sign of $k_{z}$. From now on, we shall only focus on the situation $\left(M^{2}+L^{2}\right) \gg 1$, for which

$$
\mu \approx i \sqrt{M^{2}+L^{2}} \approx i|\mu|
$$

in (16). This corresponds to either very strong electric field or highly massive field, or both. Then the general solutions for (15) are given by

$$
\varsigma_{1}\left(z_{1}\right)=C_{1} W_{\kappa_{1}, i|\mu|}\left(z_{1}\right)+D_{1} M_{\kappa_{1}, i|\mu|}\left(z_{1}\right) \text { and } \varsigma_{2}\left(z_{2}\right)=C_{2} W_{\kappa_{2}, i|\mu|}\left(z_{2}\right)+D_{2} M_{\kappa_{2}, i|\mu|}\left(z_{2}\right),
$$

where $W$ and $M$ are the Whittaker functions [56] and $C_{1}, C_{2}, D_{1}$, and $D_{2}$ are constants. 
Let us now find out the positive frequency "in" modes, i.e., the mode functions whose temporal part behaves as positive frequency plane waves as $\eta \rightarrow-\infty$. In this limit, we have [56]

$$
W_{\kappa_{1}, i|\mu|}\left(z_{1}\right) \sim e^{-2 i \eta \sqrt{k_{z}^{2}+S_{1}}} \eta^{\kappa_{1}} .
$$

Thus, for such modes, we must set $D_{1}=0=D_{2}$ in (17). Putting things together, we write the two positive frequency in mode functions as

$$
\zeta_{s, n}(\eta, \vec{x})=e^{-i H L z} e^{i \vec{k} y \cdot \vec{x}} W_{\kappa_{s}, i|\mu|}\left(z_{s}\right) h_{n}(\bar{y}) \omega_{s} \quad(s=1,2) .
$$

Likewise, since as $\eta \rightarrow 0^{-}[56]$

$$
M_{\kappa, i|\mu|}\left(z_{1}\right) \sim \eta^{i|\mu|+1 / 2},
$$

the positive frequency out modes can be defined with respect to the cosmological time $t(t=-\ln H \eta / H)$, and we choose them to be

$$
\zeta_{s, n}^{\text {out }}(\eta, \vec{x})=e^{-i H L z} e^{i \vec{k} y \cdot \vec{x}} M_{\kappa_{s}, i|\mu|}\left(z_{s}\right) h_{n}(\bar{y}) \omega_{s} \quad(s=1,2) .
$$

However, recall that the $\zeta$ 's appearing in Eqs. (18) and (19) are not the original Dirac modes, as of Eqs. (5) and (8). We thus have the complete set of positive and negative frequency in and out modes,

$U_{s, n}^{\text {in }}=\frac{1}{N_{s} a^{3 / 2}} \hat{D} \zeta_{s, n}^{\text {in }}, \quad V_{s, n}^{\text {in }}=\mathcal{C}\left(U_{s, n}^{\text {in }}\right)^{*}$,

$U_{s, n}^{\text {out }}=\frac{1}{M_{s} a^{3 / 2}} \hat{D} \zeta_{s, n}^{\text {out }}, \quad V_{s, n}^{\text {out }}=\mathcal{C}\left(U_{s, n}^{\text {out }}\right)^{*}, \quad(s=1,2)$,

where $\mathcal{C}=i \gamma^{2}$ is the charge conjugation matrix. Hence the $V$ modes appearing above are the negative frequency modes. The normalization constants appearing above are given by

$$
N_{1}=e^{\pi\left|\kappa_{1}\right| \operatorname{sgn}\left(k_{z}\right) / 2}, \quad N_{2}=e^{\pi\left|\kappa_{2}\right| \operatorname{sgn}\left(k_{z}\right) / 2}, \quad M_{1}=M_{2}=\sqrt{2|\mu|} e^{\pi|\mu| / 2},
$$

where the sign dependence of the normalization constants originates from the sign dependence (of $k_{z}$ ) of the parameters $\kappa_{s}$, (16). The explicit form of the mode functions in (20) and the evaluation of the normalization constants are discussed in the Appendix.

It is easy to check that these mode functions satisfy the orthonormality relations,

$$
\begin{aligned}
& \left(U_{s, n}^{\text {in }}\left(x ; \vec{k}_{y}\right), U_{s^{\prime}, n^{\prime}}^{\text {in }}\left(x ; \overrightarrow{k_{y}^{\prime}}\right)\right)=\left(V_{s, n}^{\text {in }}\left(x ; \vec{k}_{y}\right), V_{s \cdot n^{\prime}}^{\text {in }}\left(x ; \overrightarrow{k^{\prime}}\right)\right)=\delta^{2}\left(\vec{k}_{y}-\vec{k}_{y}^{\prime}\right) \delta_{n n^{\prime}} \delta_{s s^{\prime}}, \\
& \left(U_{s, n}^{\text {out }}\left(x ; \vec{k}_{y}\right), U_{s^{\prime}, n^{\prime}}^{\text {out }}\left(x ; \overrightarrow{k_{y}^{\prime}}\right)\right)=\left(V_{s, n}^{\text {out }}\left(x ; \vec{k}_{\jmath}\right), V_{s^{\prime}, n^{\prime}}^{\text {out }}\left(x ; \overrightarrow{k_{y}^{\prime}}\right)\right)=\delta^{2}\left(\vec{k}_{y}-\overrightarrow{k_{y}^{\prime}}\right) \delta_{n n^{\prime}} \delta_{s, s^{\prime}},
\end{aligned}
$$

with all the other inner products vanishing.

In terms of these orthonormal modes, we now make the field quantization

$$
\begin{aligned}
\psi(\eta, \vec{x}) & =\sum_{n ; s=1,2} \int \frac{d^{2} \vec{k}_{y}}{2 \pi a^{3 / 2}}\left[a_{\text {in }}\left(\vec{k}_{y}, s, n\right) U_{s, n}^{\text {in }}\left(x ; \vec{k}_{\jmath}\right)+b_{\text {in }}^{\dagger}\left(\vec{k}_{y}, s, n\right) V_{s, n}^{\text {in }}\left(x ; \vec{k}_{y}\right)\right] \\
& =\sum_{n ; s=1,2} \int \frac{d^{2} \vec{k}_{y}}{2 \pi a^{3 / 2}} \sum_{n ; s=1,2}\left[a_{\text {out }}\left(\vec{k}_{y}, s, n\right) U_{s, n}^{\text {out }}\left(x ; \vec{k}_{\jmath}\right)+b_{\text {out }}^{\dagger}\left(\vec{k}_{y}, s, n\right) V_{s, n}^{\text {out }}\left(x ; \vec{k}_{\jmath}\right)\right],
\end{aligned}
$$

where the creation and annihilation operators are assumed to satisfy the usual canonical anticommutation relations.

Using now the relations between the Whittaker functions [56],

$$
\begin{aligned}
W_{\kappa, i|\mu|}(z) & =\frac{\Gamma(-2 i|\mu|)}{\Gamma(1 / 2-i|\mu|-\kappa)} M_{\kappa, i|\mu|}(z)+\frac{\Gamma(2 i|\mu|)}{\Gamma(1 / 2+i|\mu|-\kappa)} M_{\kappa,-i|\mu|}(z), \\
M_{\kappa, i|\mu|}(z) & =-i e^{\pi|\mu|} M_{-\kappa, i|\mu|}(-z)
\end{aligned}
$$

into Eqs. (18)-(20), we find

$$
U_{s, n}^{\text {in }}\left(x ; \vec{k}_{\jmath}\right)=\frac{M_{s}}{N_{s}} \frac{\Gamma(-2 i|\mu|)}{\Gamma\left(1 / 2-i|\mu|-\kappa_{s}\right)} U_{s, n}^{\text {out }}\left(x ; \vec{k}_{\jmath}\right)+i e^{-|\mu| \pi} \frac{M_{s}}{N_{s}} \frac{\Gamma(2 i|\mu|)}{\Gamma\left(1 / 2+i|\mu|-\kappa_{s}\right)} V_{s, n}^{\text {out }}\left(x ; \vec{k}_{\jmath}\right) \quad(s=1,2) .
$$


Substituting this into (23), we obtain the Bogoliubov relations

$$
\begin{aligned}
& a_{\text {out }}\left(\vec{k}_{y}, s, n\right)=\alpha_{s} a_{\text {in }}\left(\vec{k}_{y}, s, n\right)-\beta_{s}^{*} b_{\text {in }}^{\dagger}\left(-\vec{k}_{y}, s, n\right), \\
& b_{\text {out }}\left(\vec{k}_{y}, s, n\right)=\alpha_{s} b_{\text {in }}\left(\vec{k}_{y}, s, n\right)+\beta_{s}^{*} a_{\text {in }}^{\dagger}\left(-\vec{k}_{y}, s, n\right) .
\end{aligned}
$$

The canonical anticommutation relations ensure

$$
\left|\alpha_{s}\right|^{2}+\left|\beta_{s}\right|^{2}=1 \quad(s=1,2) .
$$

Recalling we are working with $|\mu| \gg 1$, we have

$$
\beta_{s} \approx i e^{-|\mu| \pi}\left|\frac{M_{s}}{N_{s}}\right| \frac{\Gamma(2 i|\mu|)}{\Gamma\left(i|\mu|-\kappa_{s}\right)} \quad(s=1,2) .
$$

We find from the above after using some identities of the gamma function [56], the spectra of pair creation

$\left|\beta_{s}\right|_{ \pm}^{2}=e^{-\pi\left(|\mu| \pm\left|\kappa_{s}\right|\right)} \frac{\sinh \pi\left(|\mu| \pm\left|\kappa_{s}\right|\right)}{\sinh 2 \pi|\mu|} \quad(s=1,2)$,

where the \pm sign correspond, respectively, to $k_{z}>0$ and $k_{z}<0$, and originates from the fact that $\kappa_{s}$ depends upon the sign of $k_{z}$, (16). The above expression is formally similar to the case where only a background electric field is present [51]. The contribution to the particle creation from the magnetic field comes solely from the coefficients $\kappa_{s}$ and there is no contribution of it (i.e., $\kappa_{s}=0$ ) if either the electric field is vanishing or the magnetic field strength is infinitely large. Note also that, if we set $E=0$ in (28), we reproduce the well-known fermionic blackbody spectra of created particles with temperature $T_{H}=H / 2 \pi$, e.g., [30],

$$
\left|\beta_{s}\right|_{ \pm}^{2}=\frac{1}{e^{2 \pi|\mu|}+1} \quad(s=1,2)
$$

where $|\mu|=M=m / H$. The above discussions show that, in the absence of an electric field, the magnetic field cannot alter the particle creation rate, as we intuitively anticipated toward the middle of Sec. I. Finally, we also note from (28) that since $|\mu|=\left(M^{2}+L^{2}\right)^{1 / 2}$, for $E \neq 0$, and even if $B \rightarrow \infty$, the particle creation due to the electric field does not completely vanish, unlike that of the flat spacetime [40]. Once again, this should correspond to the fact that the mutual separation of the pairs created by the electric field as they propagate is also happening here due to the expanding gravitational field of the de Sitter spacetime, upon which the magnetic field has no effect.

Since the parameters $M$ and $L$ denote the dimensionless rest mass and the strength of the electric field [cf. discussion below (10)], let us consider in the following two qualitatively distinct cases, keeping in mind $\left(M^{2}+L^{2}\right) \gg 1$.

Case 1: $M^{2} \gg 1$ and $M^{2} \gg L^{2}$, hence in this case particle creation is happening chiefly due to the background spacetime curvature. We have from (28) in this limit,

$$
\left|\beta_{S}\right|_{ \pm}^{2} \approx e^{-2 \pi M}\left(1-e^{-2 \pi M} e^{\mp 2 \pi\left|\kappa_{s}\right|}+\mathcal{O}\left(e^{-4 \pi M}\right)\right) .
$$

Thus $\left|\beta_{s}\right|_{+}^{2}>\left|\beta_{s}\right|_{-}^{2}$. Since the electromagnetic field is weak here, they would have little effect on the particle creation. Note, in particular, from the expression of $\kappa_{s}$ that, if we keep the electric field strength and $k_{z}$ fixed, $\left|\beta_{s}\right|_{+}^{2}$ decreases whereas $\left|\beta_{s}\right|_{-}^{2}$ increases with the magnetic field strength, and for extremely high $B$ value, the particle creation rate coincides to that of only due to the spacetime curvature.

Case 2: $L^{2} \gg 1$ and $L^{2} \gg M^{2}$, hence in this case particle creation is happening chiefly due to the background electric field. We have from (28),

$$
\left|\beta_{s}\right|_{ \pm}^{2} \approx e^{-2 \pi L}\left(1-e^{-2 \pi L} e^{\mp 2 \pi\left|\kappa_{s}\right|}+\mathcal{O}\left(e^{-4 \pi L}\right)\right)
$$

Thus in this case also $\left|\beta_{s}\right|_{+}^{2}>\left|\beta_{s}\right|_{-}^{2}$, and $\left|\beta_{s}\right|_{+}^{2}$ decreases whereas $\left|\beta_{s}\right|_{-}^{2}$ increases with the magnetic field strength, while the other parameters are held fixed. We wish to focus only on $\left|\beta_{s}\right|_{-}^{2}$ in the following. In our computation, we shall often encounter the complex $\beta_{s}$ value. Hence, instead of using Eqs. (30) or (31), we shall work with (27), by taking numerical values of the parameters appropriate for the particular case.

Subject to the field quantization in (23), the in and the out vacua are defined as

$$
\begin{gathered}
a_{\text {in }}\left(\vec{k}_{y}, s, n\right)|0\rangle^{\text {in }}=0=b_{\text {in }}\left(\vec{k}_{y}, s, n\right)|0\rangle^{\text {in }} \quad \text { and } \\
a_{\text {out }}\left(\vec{k}_{y}, s, n\right)|0\rangle^{\text {out }}=0=b_{\text {out }}\left(\vec{k}_{y}, s, n\right)|0\rangle^{\text {out }} .
\end{gathered}
$$

Thus the Bogoliubov relations (26) imply a (normalized) squeezed state expansion between the in and out states for a given momentum,

$$
\begin{aligned}
\left|0_{k}\right\rangle^{\text {in }}= & \left(\alpha_{1}\left|0_{k}^{(1)} 0_{-k}^{(1)}\right\rangle^{\text {out }}+\beta_{1}\left|1_{k}^{(1)} 1_{-k}^{(1)}\right\rangle^{\text {out }}\right) \\
& \otimes\left(\alpha_{2}\left|0_{k}^{(2)} 0_{-k}^{(2)}\right\rangle^{\text {out }}+\beta_{2}\left|1_{k}^{(2)} 1_{-k}^{(2)}\right\rangle^{\text {out }}\right) .
\end{aligned}
$$

As we have discussed above, since we shall be working only with the "-" sign of (28), $\beta_{s}$ and $\alpha_{s}$ appearing above are understood as $\beta_{1-}$ and $\beta_{2-}$ and $\alpha_{1-}$ and $\alpha_{2-}$, respectively.

The excited in states can be written in terms of the out states by applying the in-creation operators on the left-hand side of (32) and then using the Bogoliubov relations (26) on its right-hand side. 
Finally, we note that the $s=1,2$ sectors are factorized in (32), leading to

$$
\left|0_{k}\right\rangle^{\text {in }}=\left|0_{k}^{(1)}\right\rangle^{\text {in }} \otimes\left|0_{k}^{(2)}\right\rangle^{\text {in }} .
$$

Thus, for simplicity, we can work only with a single sector, say $\left|0_{k}^{(1)}\right\rangle^{\text {in }}$, of the in vacuum.

Being equipped with these, we are now ready to go to the computation of the Bell violation. However, before we do that, we wish to compute the entanglement entropy associated with the vacuum state.

\section{ENTANGLEMENT ENTROPY OF THE VACUUM}

If a system is made of two subsystems, say $A$ and $B$, the entanglement entropy of $A$ is defined as the von Neumann entropy of $\rho_{A}, S\left(\rho_{A}\right)=-\operatorname{Tr}_{A}\left(\rho_{A} \ln \rho_{A}\right)$, where $\rho_{A}$ is the reduced density operator $\rho_{A}=\operatorname{Tr}_{B} \rho_{A B}$. The entanglement entropy of $B$ is defined in a likewise manner. If $\rho_{A B}$ corresponds to a pure state, one has $S\left(\rho_{A}\right)=S\left(\rho_{B}\right)$, and it is vanishing when $\rho_{A B}$ is also separable, $\rho_{A B}=$ $\rho_{A} \otimes \rho_{B}$. The von Neumann entropies satisfy a subadditivity $S\left(\rho_{A B}\right) \leq S\left(\rho_{A}\right)+S\left(\rho_{B}\right)$, where $S\left(\rho_{A B}\right)$ is the von Neumann entropy corresponding to $\rho_{A B}$, and the equality holds if and only if $\rho_{A B}$ is separable, e.g., [16].

We wish to compute the entanglement entropy for the state $\left|0_{k}^{(1)}\right\rangle^{\text {in }}$, defined at the end of the preceding section. The density matrix corresponding to this state is pure, $\rho_{0}=\left|0_{k}^{(1)}\right\rangle^{\text {inin }}\left\langle 0_{k}^{(1)}\right|$. Using (32), we write down $\rho_{0}$ in terms of the out states, which contain both $k$ and $-k$ degrees of freedom. The reduced density matrix corresponding to the $k$ sector (say, particle) is given by $\rho_{k}=\operatorname{Tr}_{-k} \rho_{0}=$ $\left|\alpha_{1}\right|^{2}\left|0_{k}^{(1)}\right\rangle^{\text {outout }}\left\langle\left. 0_{k}^{(1)}|+| \beta_{1}\right|^{2} \mid 1_{k}^{(1)}\right\rangle^{\text {outout }}\left\langle 1_{k}^{(1)}\right|$, and hence the entanglement entropy is give by

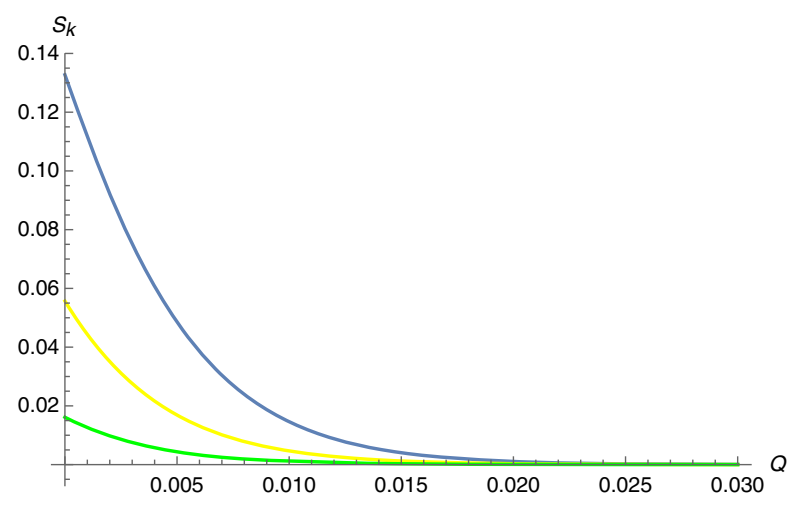

$$
S_{k}=-\operatorname{Tr}_{k} \rho_{k} \ln \rho_{k}=-\left[\ln \left(1-\left|\beta_{1}\right|^{2}\right)+\left|\beta_{1}\right|^{2} \ln \frac{\left|\beta_{1}\right|^{2}}{1-\left|\beta_{1}\right|^{2}}\right] .
$$

We also have $S_{k}=S_{-k}$, as we are dealing with a pure state.

Since we are chiefly interested here in the effect of the magnetic field strength, let us extract a dimensionless parameter from (16),

$$
Q=\frac{2 e n B}{k_{z}^{2}} .
$$

The $Q$ dependence of $S_{k}$ is depicted in Fig. 1 for the two cases ["large" $\left(L^{2} \gg M^{2}\right)$ and "small" $\left(L^{2} \ll M^{2}\right)$ electric fields], discussed in the preceding section. For a given mode (i.e., $n, k_{z}$ fixed) thus, the increase in $Q$ corresponds to the increase in the $B$ value. As can be seen in the figure, the entanglement entropy decreases monotonically with the increase in the magnetic field strength. This corresponds to the fact that the vacuum entanglement entropy originates from the pair creation, which decreases with increasing $B$ for both the cases we have considered.

\section{THE VIOLATION OF THE BELL INEQUALITIES}

\section{A. The Bell inequalities}

The construction of the Bell or the Bell-MerminKlyshko operators for fermions are similar to that of the scalar field, e.g., $[16,25]$ and references therein. Let us consider two pairs of noncommuting observables defined, respectively, over the Hilbert spaces $\mathcal{H}_{A}$ and $\mathcal{H}_{B}:\left(O_{1}, O_{1}^{\prime}\right) \in \mathcal{H}_{A}$ and $\left(O_{2}, O_{2}^{\prime}\right) \in \mathcal{H}_{B}$. We assume that these are spin- $1 / 2$ operators along specific directions, such as $O=n_{i} \sigma_{i}, O^{\prime}=n_{i}^{\prime} \sigma_{i}$, where $\sigma_{i}$ 's are the Pauli matrices and $n_{i}, n_{i}^{\prime}$ are unit vectors on the three-dimensional

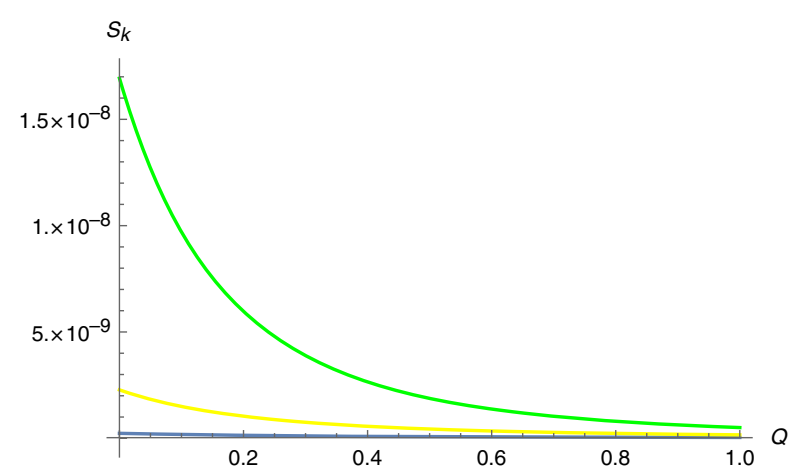

FIG. 1. We have plotted entanglement entropy (33) corresponding to the vacuum state with respect to the parameter $Q=2 n e B / k_{z}^{2}$. The left plot corresponds to the case $L^{2} \gg M^{2}$, where we have taken $L=100$ and different curves correspond to different $M$ values (blue $M=10$, yellow $M=12$, and green $M=16$ ). The right plot corresponds to $M^{2} \gg L^{2}$, where we have taken $M=5$, and different curves correspond to different $L$ values (blue $L=1$, yellow $L=1.5$, and green $L=2$ ). For a given mode, the entanglement entropy decreases monotonically with increasing $B$ for both the cases due to the decrease in the particle creation. See main text for detail. 
Euclidean space. The eigenvalues of each of these operators are \pm 1 . The Bell operator $\mathcal{B} \in \mathcal{H}_{A} \otimes \mathcal{H}_{B}$ is defined as (suppressing the tensor product sign)

$$
\mathcal{B}=O_{1}\left(O_{2}+O_{2}^{\prime}\right)+O_{1}^{\prime}\left(O_{2}-O_{2}^{\prime}\right) .
$$

In theories with classical local hidden variables, we have the so-called Bell's inequality, $\left\langle\mathcal{B}^{2}\right\rangle \leq 4$ and $|\langle\mathcal{B}\rangle| \leq 2$. However, this inequality is violated in quantum mechanics as follows. We have from (34),

$$
\mathcal{B}^{2}=\mathbf{I}-\left[O_{1}, O_{1}^{\prime}\right]\left[O_{2}, O_{2}^{\prime}\right],
$$

where $\mathbf{I}$ is the identity operator. Using the commutation relations for the Pauli matrices, one gets $|\langle\mathcal{B}\rangle| \leq 2 \sqrt{2}$, thereby obtaining a violation of Bell's inequality, where the equality is regarded as the maximum violation.

The above construction can be extended to multipartite systems with pure density matrices as well, corresponding to squeezed states formed by mixing different modes. We refer our readers to [25] and references therein for details.

We wish to investigate below Bell's inequality violation for the vacuum, as well as some maximally entangled initial states.

\section{B. Bell violation for the vacuum state}

We wish to find out the expectation value of $\mathcal{B}$, (34), with respect to the vacuum state $\left|0_{k}^{(1)}\right\rangle$ in, given at the end of Sec. II. In order to do this, one usually introduces pseudospin operators measuring the parity in the Hilbert space along different axes, e.g., [25] and references therein. These operators for fermionic systems with eigenvalues \pm 1 are defined as

$$
\hat{\mathbf{n}} . \mathbf{S}=S_{z} \cos \theta+\sin \theta\left(e^{i \phi} S_{-}+e^{-i \phi} S_{+}\right),
$$

where $\hat{\mathbf{n}}=(\sin \theta \cos \phi, \sin \theta \sin \phi, \cos \phi)$ is a unit vector in the Euclidean three-plane. The action of the operators $S_{z}$ and $S_{ \pm}$are defined on the out states,

$$
\begin{aligned}
& S_{z}|0\rangle=-|0\rangle, \quad S_{z}|1\rangle=|1\rangle, \quad S_{+}|0\rangle=|1\rangle, \\
& S_{+}|1\rangle=0, \quad S_{-}|0\rangle=0, \quad S_{-}|1\rangle=|0\rangle .
\end{aligned}
$$

Without any loss of generality, we take the operators to be confined to the $x-z$ plane, so that we may set $\phi=0$ in (36). We may then take in (34), $O_{i}=\hat{\mathbf{n}}_{i} \cdot \mathbf{S}$ and $O_{i}^{\prime}=\hat{\mathbf{n}}_{i}^{\prime} \cdot \mathbf{S}$ with $i=1,2$. Here $\hat{\mathbf{n}}_{i}$ and $\hat{\mathbf{n}}_{i}^{\prime}$ are two pairs of unit vectors in the Euclidean three-plane, characterized by their angles with the $z$ axis, $\theta_{i}$ and $\theta_{i}^{\prime}$ (with $i=1,2$ ), respectively.

Using the above constructions and the squeezed state expansion [(32)] and also the operations (37) defined on the out states, the desired expectation value is given by

$$
\begin{aligned}
{ }_{\text {in }}\left\langle 0_{k}^{(1)}|\mathcal{B}| 0_{k}^{(1)}\right\rangle^{\text {in }}= & {\left[E\left(\theta_{1}, \theta_{2}\right)+E\left(\theta_{1}, \theta_{2}^{\prime}\right)+E\left(\theta_{1}^{\prime}, \theta_{2}\right)\right.} \\
& \left.-E\left(\theta_{1}^{\prime}, \theta_{2}^{\prime}\right)\right],
\end{aligned}
$$

where $O_{i}$ and $O_{i}^{\prime}$ are assumed to operate, respectively, on the $k$ and $-k$ sectors of the out states in (32), and

$$
E\left(\theta_{1}, \theta_{2}\right)=\cos \theta_{1} \cos \theta_{2}+2\left|\alpha_{1} \beta_{1}\right| \sin \theta_{1} \sin \theta_{2} .
$$

Choosing now $\theta_{1}=0, \theta_{1}^{\prime}=\pi / 2$, and $\theta_{2}=-\theta_{2}^{\prime}$, we have from (38),

$$
{ }^{\text {in }}\left\langle 0_{k}^{(1)}|\mathcal{B}| 0_{k}^{(1)}\right\rangle \text { in }=2\left(\cos \theta_{2}+2\left|\alpha_{1} \beta_{1}\right| \sin \theta_{2}\right) .
$$

The above expression maximizes at $\theta_{2}=\tan ^{-1}\left(2\left|\alpha_{1} \beta_{1}\right|\right)$, so that the above expectation value becomes

$$
\langle\mathcal{B}\rangle_{\max }=2\left(1+4\left|\alpha_{1} \beta_{1}\right|^{2}\right)^{1 / 2} .
$$

Thus $\langle\mathcal{B}\rangle_{\max } \geq 2$, and hence there is Bell violation for $\left|\beta_{1}\right|>0$. We have plotted $\langle\mathcal{B}\rangle_{\max }$ in Fig. 2 with respect to the parameter $Q=2 e n B / k_{z}^{2}$ as earlier. We have considered only the case of strong electric field $L^{2} \gg M^{2}$, for the other case does not show any significant violation nor numerical variation. As for the vacuum entanglement entropy, Fig. 1, the Bell violation decreases monotonically with the increasing magnetic field (for a given mode) and reaches the value 2. Once again, this happens due to the suppression of the particle creation by the magnetic field.

Note that the vacuum state is pure. Instead of vacuum, if we consider a pure but maximally entangled state, make its squeezed state expansion, and then trace out some parts of it in order to construct a bipartite subsystem, the resulting density matrix turns out to be mixed. The above

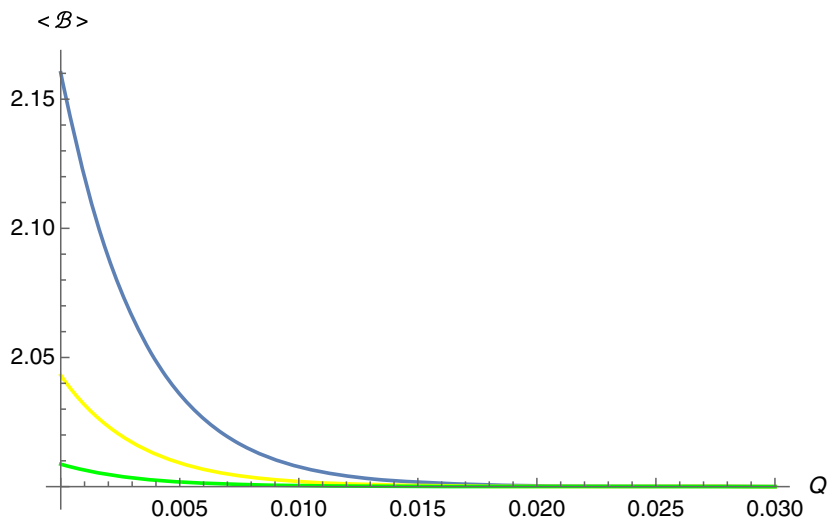

FIG. 2. We have plotted Bell's violation $\langle\mathcal{B}\rangle_{\max }[(39)]$, corresponding to the vacuum state, with respect to the parameter $Q=2 e n B / k_{z}^{2}$. We have plotted only the case $L^{2} \gg M^{2}$, for the other case $\left(M^{2} \gg L^{2}\right)$ does not show any significant violation or numerical variation. We have taken $L=100$ and different curves correspond to different $M$ values (blue $M=10$, yellow $M=12$, and green $M=16$ ). 
construction is valid for pure ensembles only and one requires a different formalism to deal with mixed ensembles, e.g., [6,21]. We wish to study two such cases below, in order to demonstrate their qualitative differences with the vacuum case.

\section{Bell violation for maximally entangled initial states}

We wish to consider maximally entangled initial states (corresponding to two fermionic fields) in the following. For computational simplicity, we assume that both fields have the same rest mass, and we consider modes in which their momenta along the $z$ direction and the Landau levels are the same.

The density matrix corresponding to the initial state can be expanded into the out states via (32) and then any 2 degrees of freedom is traced out in order to construct a bipartite system. The resulting reduced density matrix turns out to be mixed. For such a system, the Bell violation measure is defined as $[6,21]$

$$
\left\langle\mathcal{B}_{\max }\right\rangle=2 \sqrt{\lambda_{1}+\lambda_{2}}
$$

where $\lambda_{1}$ and $\lambda_{2}$ are the two largest eigenvalues of the $3 \times 3$ matrix $U=\left(T_{\rho}\right)^{\mathrm{T}} T_{\rho}$, with $T \equiv \operatorname{Tr}\left[\rho \sigma_{i} \otimes \sigma_{j}\right]$, where $\rho$ is the aforementioned mixed density matrix. $T$ is called the correlation matrix for the generalized Bloch decomposition of $\rho$. Since the reduced density matrix represents a bipartite system, the violation of the Bell inequality as earlier will correspond to $\left\langle\mathcal{B}_{\max }\right\rangle>2$ in (40).

We begin by considering the initial state,

$$
|\psi\rangle=\frac{\left|0_{p} 0_{-p} 0_{k} 0_{-k}\right\rangle^{\text {in }}+\left|1_{p} 0_{-p} 0_{k} 1_{-k}\right\rangle^{\text {in }}}{\sqrt{2}} .
$$

In the four entries of a ket above, the first pair of states corresponds to one fermionic field, whereas the last pair corresponds to another. The \pm sign in front of the momenta stands, respectively, for the particle and antiparticle degrees of freedom.

Recall that we are assuming the created particles have the same rest mass, and we are working with modes for which the Landau levels and the $k_{z}$ values for both the fields are coincident. Using then Eqs. (26) and (32), we reexpress (41) in the out basis as

$$
|\psi\rangle=\frac{\left(\alpha_{1}\left|0_{p} 0_{-p}\right\rangle^{\text {out }}+\beta_{1}\left|1_{p} 1_{-p}\right\rangle^{\text {out }}\right)\left(\alpha_{1}\left|0_{k} 0_{-k}\right\rangle^{\text {out }}+\beta_{1}\left|1_{k} 1_{-k}\right\rangle^{\text {out }}\right)+\left|0_{p} 1_{-p}\right\rangle^{\text {out }}\left|1_{k} 0_{-k}\right\rangle^{\text {out }}}{\sqrt{2}}
$$

We shall focus below only on the correlations between the particle-particle and the particle-antiparticle sectors corresponding to the density matrix of the above state. Accordingly, tracing out first the antiparticle-antiparticle degrees of freedom of the density matrix $\rho^{(0)}=|\psi\rangle\langle\psi|$, we construct the reduced density matrix for the particle-particle sector,

$$
\rho_{k, p}^{0}=\operatorname{Tr}_{-k,-p}\left(\rho^{(0)}\right)=\frac{1}{2}\left(\begin{array}{cccc}
\left|\alpha_{1}\right|^{4} & 0 & 0 & 0 \\
0 & \left|\alpha_{1} \beta_{1}\right|^{2} & \left(\alpha_{1} \beta_{1}\right)^{*} & 0 \\
0 & \alpha_{1} \beta_{1} & \left|\alpha_{1} \beta_{1}\right|^{2} & 0 \\
0 & 0 & 0 & \left|\beta_{1}\right|^{4}
\end{array}\right)
$$

Likewise, we can obtain the reduced density matrix for the particle-antiparticle sector,

$$
\rho_{p,-k}^{0}=\operatorname{Tr}_{-p, k}\left(\rho^{(0)}\right)=\frac{1}{2}\left(\begin{array}{cccc}
\left|\alpha_{1}\right|^{4} & 0 & 0 & \left(\alpha_{1}^{*}\right)^{2} \\
0 & \left|\alpha_{1} \beta_{1}\right|^{2} & 0 & 0 \\
0 & 0 & \left|\alpha_{1} \beta_{1}\right|^{2} & 0 \\
\alpha_{1}^{2} & 0 & 0 & \left|\beta_{1}\right|^{4}+1
\end{array}\right) .
$$

The correlation matrices corresponding to Eqs. (43) and (44) are, respectively, given by

$$
T\left(\rho_{k, p}^{0}\right)=\left(\begin{array}{ccc}
\operatorname{Re}\left(\alpha_{1} \beta_{1}\right) & 0 & 0 \\
0 & -\operatorname{Re}\left(\alpha_{1} \beta_{1}\right) & 0 \\
0 & 0 & \frac{1}{2}\left(\left|\alpha_{1}\right|^{4}-2\left|\alpha_{1}\right|^{2}\left|\beta_{1}\right|^{2}-1+\left|\beta_{1}\right|^{4}\right)
\end{array}\right)
$$

and 

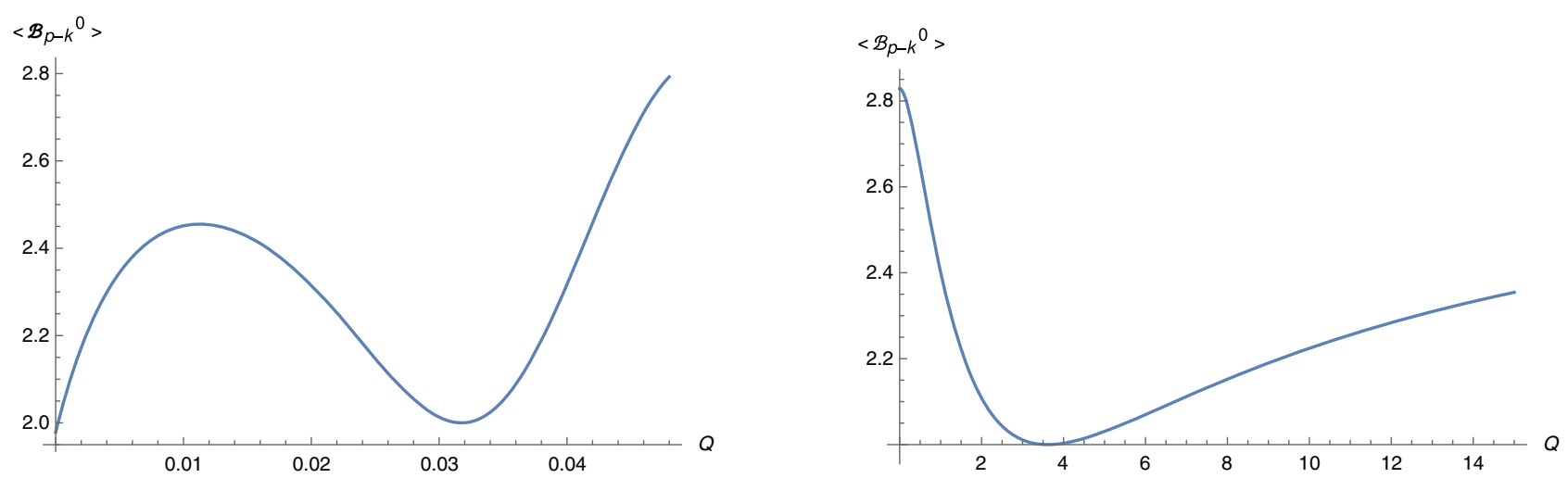

FIG. 3. Bell violation for the particle-antiparticle sector $\rho_{p,-k}^{0},(44)$, corresponding to the initial state in (41). We have plotted (48) with respect to the parameter $Q=2 n e B / k_{z}^{2}$. The left plot corresponds to $L^{2} \gg M^{2}(L=100$ and $M=10)$, whereas the right one corresponds to $M^{2} \gg L^{2}(M=5$ and $L=1)$. $\left\langle\mathcal{B}_{p-k}^{0}\right\rangle_{\max }>2$ corresponds to the Bell violation.

$$
T\left(\rho_{p,-k}^{0}\right)=\left(\begin{array}{ccc}
\operatorname{Re}\left(\alpha_{1}^{2}\right) & 0 & 0 \\
0 & \operatorname{Re}\left(\alpha_{1}^{2}\right) & 0 \\
0 & 0 & \frac{1}{2}\left(\left|\alpha_{1}\right|^{4}-2\left|\alpha_{1}\right|^{2}\left|\beta_{1}\right|^{2}+1+\left|\beta_{1}\right|^{4}\right)
\end{array}\right) .
$$

Using Eqs. (45) and (46), we compute the matrices $U\left(\rho_{k p}^{0}\right)=$ $\left(T\left(\rho_{k, p}^{0}\right)\right)^{\mathrm{T}} T\left(\rho_{k, p}^{0}\right)$ and $U\left(\rho_{p,-k}^{0}\right)=\left(T\left(\rho_{p,-k}^{0}\right)^{\mathrm{T}} T\left(\rho_{p,-k}^{0}\right)\right.$. Equation (40) yields then after a little algebra

$$
\left\langle\mathcal{B}_{k p}^{0}\right\rangle_{\max }=2 \sqrt{2} \operatorname{Re}\left(\alpha_{1} \beta_{1}\right)
$$

and

$$
\left\langle\mathcal{B}_{p-k}^{0}\right\rangle_{\max }=2 \sqrt{\left(\operatorname{Re}\left(\alpha_{1}^{2}\right)\right)^{2}+\left(1-2\left|\alpha_{1} \beta_{1}\right|^{2}\right)^{2}} .
$$

We have plotted $\left\langle\mathcal{B}_{p-k}^{0}\right\rangle_{\max }$ in Fig. 3 with respect to the parameter $Q=2 n e B / k_{z}^{2}$ as earlier, depicting the Bell violation $\left(\left\langle\mathcal{B}_{p-k}^{0}\right\rangle_{\max }>2\right)$ for both strong and weak electric fields. $\left\langle\mathcal{B}_{k p}^{0}\right\rangle_{\max }$, on the other hand, does not show any such violation.

We next consider another maximally entangled state given by

$$
|\chi\rangle=\frac{\left|1_{p} 0_{-p} 0_{k} 0_{-k}\right\rangle^{\text {in }}+\left|0_{p} 0_{-p} 1_{k} 0_{-k}\right\rangle^{\text {in }}}{\sqrt{2}} .
$$

Following similar steps as described above, by partially tracing out the original density matrix $\rho^{(1)}=|\chi\rangle\langle\chi|$, we have the mixed bipartite density matrices, respectively, for the particle-particle and the particle-antiparticle sectors,

$\rho_{k, p}^{1}=\operatorname{Tr}_{-k,-p}\left(\rho^{(1)}\right) \quad$ and $\quad \rho_{p,-k}^{1}=\operatorname{Tr}_{-p, k}\left(\rho^{(1)}\right)$,

which, respectively, yield the Bell violations
$\left\langle\mathcal{B}_{k p}^{1}\right\rangle_{\max }=2 \sqrt{2}\left|\alpha_{1}\right|^{2} \quad$ and $\quad\left\langle\mathcal{B}_{p-k}^{1}\right\rangle_{\max }=2 \sqrt{2} \operatorname{Re}\left(\beta_{1} \alpha_{1}^{*}\right)$.

We have plotted $\left\langle\mathcal{B}_{k p}^{1}\right\rangle_{\max }$ in Fig. 4 with respect to the parameter $Q$ for strong electric field $L^{2} \gg M^{2}$. For $M^{2} \gg L^{2}$, we also have violation, however, it does not show any significant numerical variation. On the other hand, we find no violation for the particle-antiparticle sector $\left\langle\mathcal{B}_{p-k}^{1}\right\rangle_{\max }$.

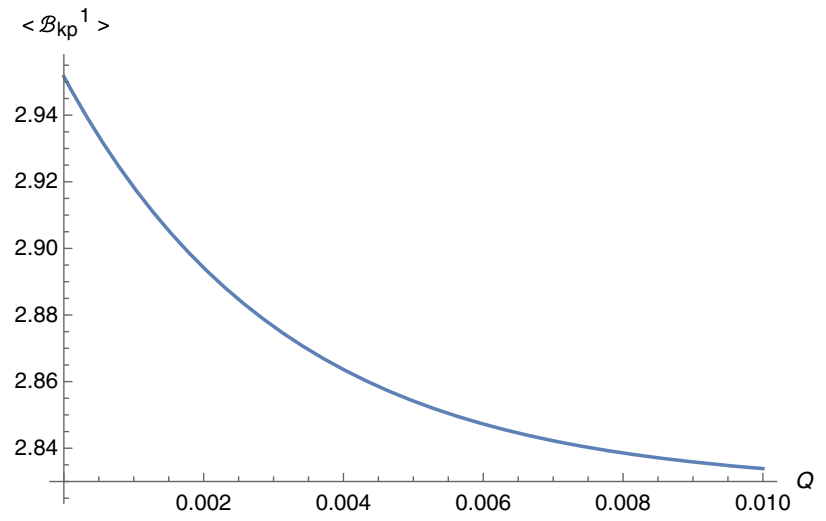

FIG. 4. Bell violation for $\rho_{k, p}^{1},(50)$, corresponding to the initial state in (49). We have plotted $\left\langle\mathcal{B}_{k p}^{1}\right\rangle,(51)$, with respect to the parameter $Q=2 n e B / k_{z}^{2}$, for $L^{2} \gg M^{2}(L=100$ and $M=10)$. The other case, $M^{2} \gg L^{2}$, does show Bell violation but there is no significant numerical variation. Note that in contrast to Fig. 3 the behavior is monotonic here and qualitatively it rather resembles the vacuum case, as in Fig. 2. 
Before we conclude, we wish to further extend the above results for the so-called fermionic $\alpha$ vacua.

\section{THE CASE OF THE FERMIONIC $\alpha$ VACUA}

The fermionic $\alpha$ vacua, like the scalar field [57,58], correspond to a Bogoliubov transformation characterized by a parameter $\alpha$ in the in mode field quantization. Although such vacua may not be very useful to do perturbation theory, e.g., $[59,60]$, it still attracts attention chiefly from the perspective of the so-called transPlanckian censorship conjecture, e.g., [61].

In order to construct such vacua, from (23), we define a new set of annihilation and creation operators [62],

$c^{\alpha}\left(\vec{k}_{y}, s, n\right)=\cos \alpha a_{\mathrm{in}}\left(\vec{k}_{y}, s, n\right)-\sin \alpha b_{\mathrm{in}}^{\dagger}\left(\vec{k}_{y}, s, n\right)$,

$d^{\alpha}\left(\vec{k}_{y}, s, n\right)=\cos \alpha b_{\text {in }}\left(\vec{k}_{y}, s, n\right)+\sin \alpha a_{\text {in }}^{\dagger}\left(\vec{k}_{y}, s, n\right)$,

where the parameter $\alpha$ is real and $0 \leq \alpha \leq \pi / 2$. The above relations indicate that we need to define a new, one parameter family of vacuum state $|0\rangle_{\alpha}$, so that

$$
c^{\alpha}|0\rangle_{\alpha}=0=d^{\alpha}|0\rangle_{\alpha} .
$$

An $\alpha$-vacuum state is related to the original in-vacuum state via a squeezed state expansion. Note that (52) does not mix the sectors $s=1$ and $s=2$. Thus, as in the previous analysis, we work only with the $s=1$ sector and write for the normalized $\alpha$-vacuum state,

$$
\left|0_{k}\right\rangle_{\alpha}^{(1)}=\cos \alpha\left|0_{k}^{(1)} 0_{-k}^{(1)}\right\rangle^{\text {in }}+\sin \alpha\left|1_{k}^{(1)} 1_{-k}^{(1)}\right\rangle^{\text {in }} .
$$

Using now (32) into the above equation, we reexpress $|0\rangle_{\alpha}^{(1)}$ in terms of the out states

$$
\left|0_{k}\right\rangle_{\alpha}^{(1)}=\alpha^{\prime}\left|0_{k}^{(1)} 0_{-k}^{(1)}\right\rangle^{\text {out }}+\beta^{\prime}\left|1_{k}^{(1)} 1_{-k}^{(1)}\right\rangle^{\text {out }},
$$

where

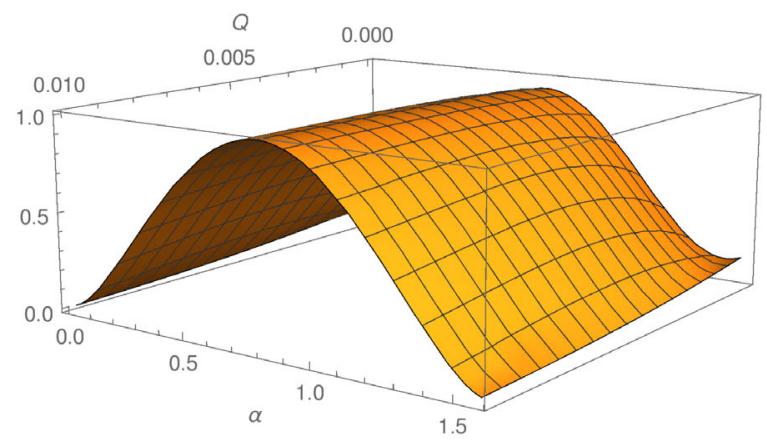

$$
\begin{aligned}
& \alpha^{\prime}=\frac{\alpha_{1} \cos \alpha+\beta_{1} \sin \alpha}{\sqrt{1+2\left(\alpha_{1} \beta_{1}^{*}+\beta_{1} \alpha_{1}^{*}\right) \cos \alpha \sin \alpha}}, \\
& \beta^{\prime}=\frac{\alpha_{1} \sin \alpha+\beta_{1} \cos \alpha}{\sqrt{1+2\left(\alpha_{1} \beta_{1}^{*}+\beta_{1} \alpha_{1}^{*}\right) \cos \alpha \sin \alpha}}
\end{aligned}
$$

are the effective Bogoliubov coefficients. Note the formal similarity between Eqs. (54) and (32). Setting $\alpha=0$ in the first reproduces the second.

The above-mentioned formal similarity thus ensures that the expressions for either the vacuum entanglement entropy or the Bell violation for the $\alpha$ states can be obtained from our earlier results [Eqs. (33), (39), (47), (48), and (51)], by simply making the replacements

$$
\alpha_{1} \rightarrow \alpha_{1}^{\prime}, \quad \text { and } \quad \beta_{1} \rightarrow \beta_{1}^{\prime} .
$$

Some aspects of entanglement for scalar $\alpha$ vacua can be seen in, e.g., [63-66] (also references therein). See also [28] for discussion on the natural emergence of $\alpha$-like vacua for fermions in the hyperbolic de Sitter spacetime.

For the fermionic case, the vacuum entanglement entropy, (33), modifies to the $\alpha$ vacua as

$$
S_{k}^{\alpha}=-\left[\ln \left(1-\left|\beta_{1}^{\prime}\right|^{2}\right)+\left|\beta_{1}^{\prime}\right|^{2} \ln \frac{\left|\beta_{1}^{\prime}\right|^{2}}{1-\left|\beta_{1}^{\prime}\right|^{2}}\right],
$$

which is plotted in Fig. 5 with respect to the parameters $Q=2 e n B / k_{z}^{2}$ and $\alpha$. We see that $S_{k}^{\alpha}$ first increases with increase in the parameter $\alpha$ and has its maximum at $\alpha=\pi / 4$, after which it decreases and becomes vanishing as $\alpha \rightarrow \pi / 2$. Likewise, the Bell violation for the vacuum state, (39), can be extended to the $\alpha$ vacua and is plotted in Fig. 6. Like the vacuum entanglement entropy, the vacuum Bell violation also reaches maximum at $\alpha=\pi / 4$ and becomes vanishing as $\alpha \rightarrow \pi / 2$.

The vanishing of both vacuum entanglement entropy and Bell violation as $\alpha \rightarrow \pi / 2$ can be understood as follows. In this limit, only the excited state part of (53) survives.

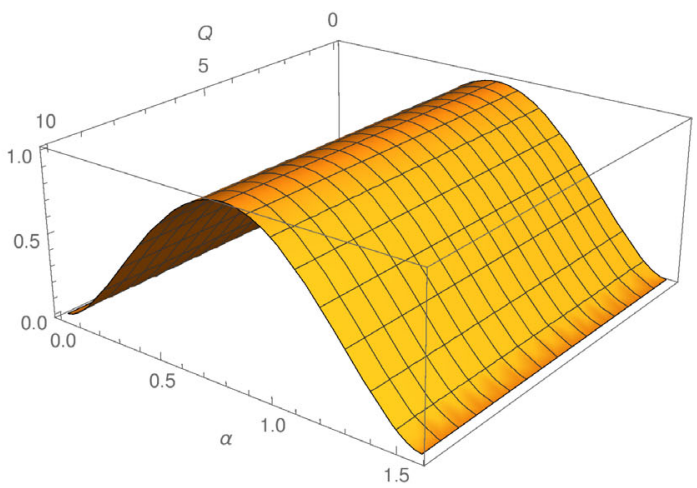

FIG. 5. Entanglement entropy for the fermionic $\alpha$ vacua, (53). We have plotted (56) vs the parameters $Q=2 e n B / k_{z}^{2}$ and $\alpha$. The left one corresponds to $L^{2} \gg M^{2}(L=100$ and $M=10)$, whereas the right one corresponds to $M^{2} \gg L^{2}(M=5$ and $L=1)$. See main text for discussions. 

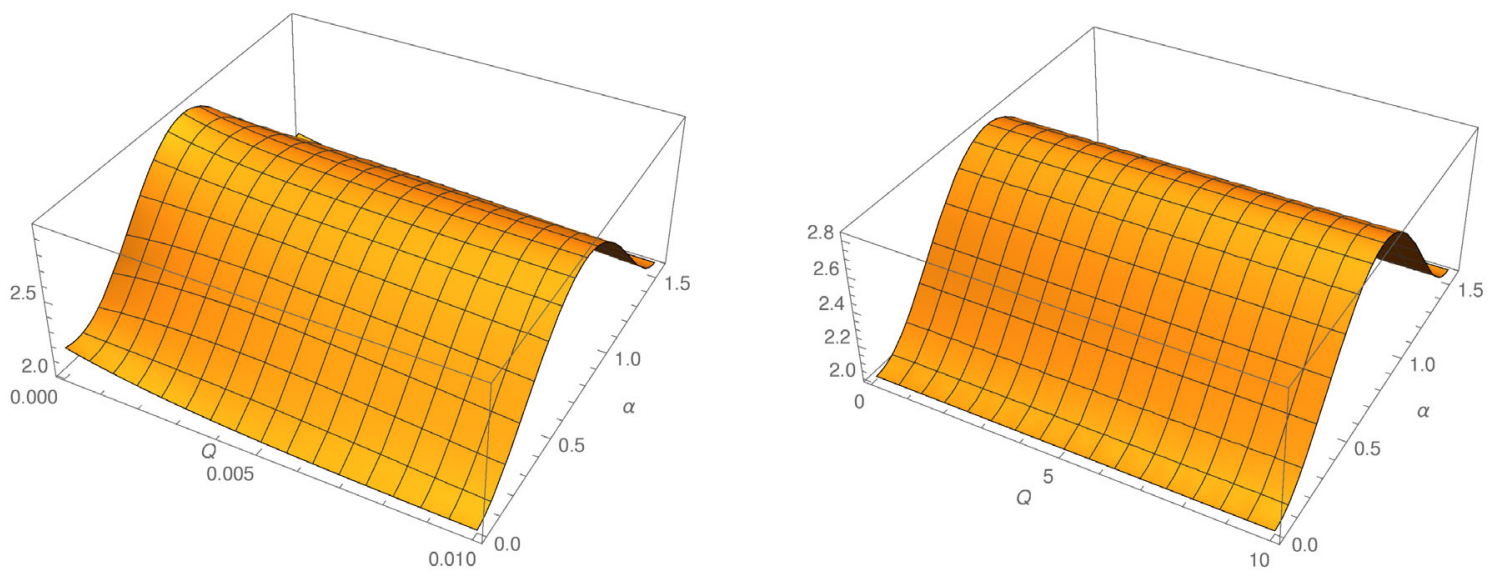

FIG. 6. Bell violation for the fermionic $\alpha$ vacua. As we have discussed in the main text, we have plotted (39) after replacing $\alpha_{1}$ and $\beta_{1}$, respectively, by $\alpha_{1}^{\prime}$ and $\beta_{1}^{\prime}$ given by (55). The left one corresponds to $L^{2} \gg M^{2}(L=100$ and $M=10)$, whereas the right one corresponds to $M^{2} \gg L^{2}(M=5$ and $L=1)$. $\langle\mathcal{B}\rangle_{\alpha, \max }>2$ corresponds to the Bell violation.
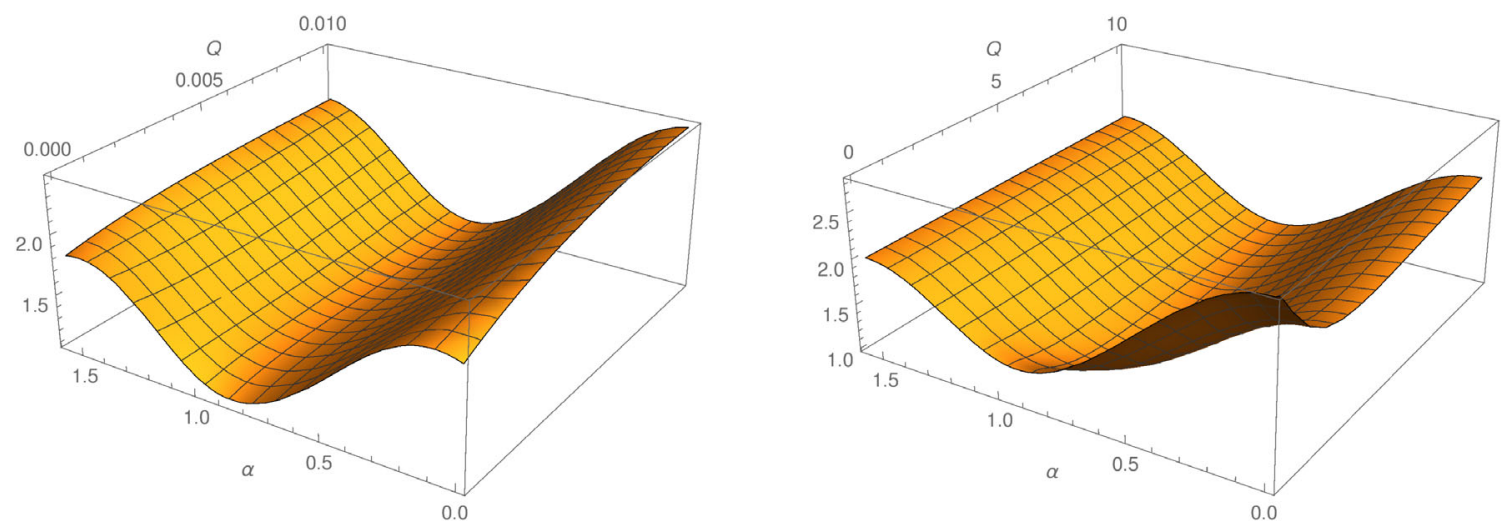

FIG. 7. Bell violation for the particle-antiparticle sector corresponding to the initial $\alpha$ state in (57). As we have discussed in the main text, we have basically plotted (48) after replacing $\alpha_{1}, \beta_{1}$, respectively, by $\alpha_{1}^{\prime}$ and $\beta_{1}^{\prime}$ [(55)]. The left plot corresponds to $L^{2} \gg M^{2}$ ( $L=100$ and $M=10$ ), whereas the right one corresponds to $M^{2} \gg L^{2}(M=5$ and $L=1)$. $\langle\mathcal{B}\rangle_{\alpha, \max }>2$ corresponds to the Bell violation. The particle-particle sector corresponding to this initial state does not show any Bell violation, like the $\alpha=0$ case discussed in Sec. IV C.
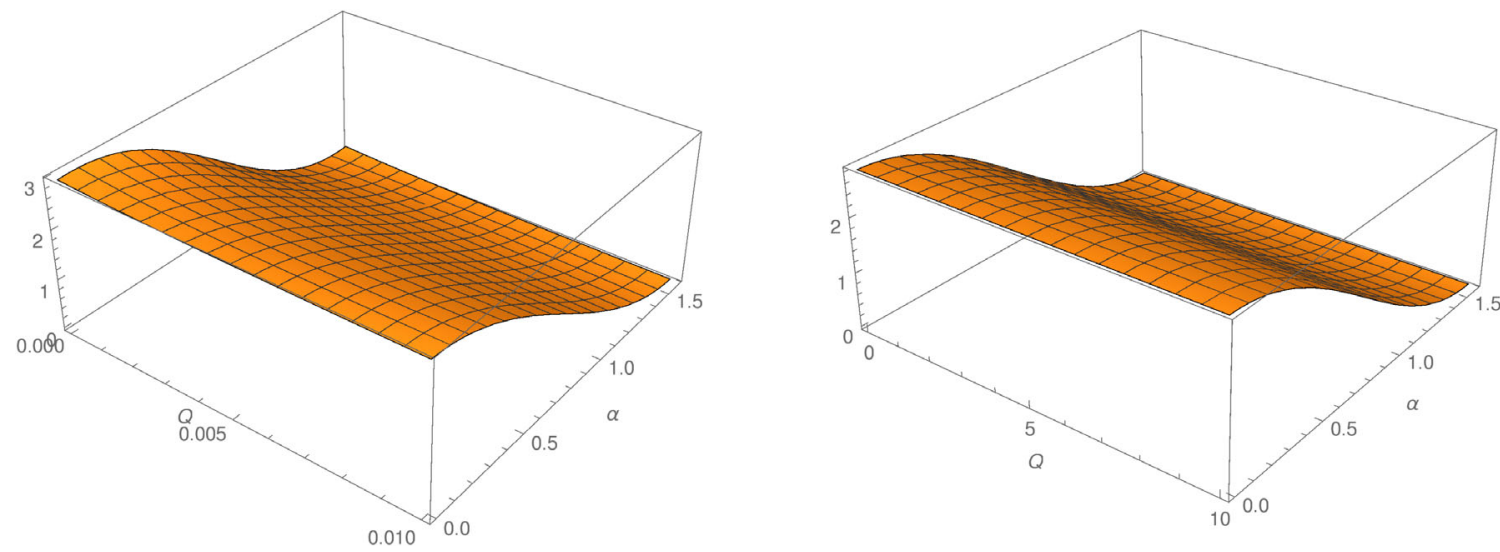

FIG. 8. Bell violation for the particle-particle sector corresponding to the initial state in (58). The left plot corresponds to $L^{2} \gg M^{2}$ ( $L=100$ and $M=10)$, whereas the right one corresponds to $M^{2} \gg L^{2}(M=5$ and $L=1)$. As earlier, $\langle\mathcal{B}\rangle_{\alpha, \max }>2$ corresponds to the Bell violation. The particle-antiparticle sector corresponding to this initial state does not show any violation, like the $\alpha=0$ case discussed in Sec. IV C. 
Equation (32) then implies that the corresponding out-basis expansion of this state is not only pure, but also separable. Thus, in this limit, no entanglement survives.

Let us now come to the case of the maximally entangled states. The states of Eqs. (41) and (49), respectively, modify as

$$
|\psi\rangle_{\alpha}=\frac{\left|0_{p} 0_{-p} 0_{k} 0_{-k}\right\rangle_{\alpha}^{\text {in }}+\left|1_{p} 0_{-p} 0_{k} 1_{-k}\right\rangle_{\alpha}^{\text {in }}}{\sqrt{2}}
$$

and

$$
|\chi\rangle_{\alpha}=\frac{\left|1_{p} 0_{-p} 0_{k} 0_{-k}\right\rangle_{\alpha}^{\text {in }}+\left|0_{p} 0_{-p} 1_{k} 0_{-k}\right\rangle_{\alpha}^{\text {in }}}{\sqrt{2}} .
$$

Using (53), and the method described in Sec. IV C, we can easily extend the results of the Bell violation we found earlier. As we mentioned earlier, this generalization effectively corresponds to just replacing $\alpha_{1}, \beta_{1}$, respectively, by $\alpha_{1}^{\prime}$ and $\beta_{1}^{\prime}[(55)]$ in appropriate places [e.g., in (47)]. We have plotted these Bell violations in Figs. 7 and 8 .

\section{SUMMARY AND OUTLOOK}

In this work, we have discussed the fermionic Bell violation in the cosmological de Sitter spacetime, in the presence of primordial electromagnetic fields of constant strengths. We have found relevant in and out orthonormal Dirac mode functions, the Bogoliubov coefficients and the resultant squeezed state relationship between the in and out states, in Sec. II. Using these key results, we have computed the vacuum entanglement entropy and the Bell violation (for both vacuum and two maximally entangled initial states), respectively, in Secs. III and IV. These results are extended further to the so-called fermionic $\alpha$ vacua in Sec. V. We have focused on two qualitatively distinct cases here - the "strong" electric filed and the "heavy" mass limits (with respect to the Hubble constant), cf. Eqs. (30) and (31).

As we have discussed in Sec. I, a background magnetic field alone cannot create vacuum instability, but in the presence of spacetime curvature and electric field, it can affect such instability or the rate of the particle pair creation. This is manifest from (28), which receives, as we have discussed, no contribution from the magnetic field if the electric field strength is vanishing. Whereas if the magnetic field strength is very large compared to that of the electric field, the particle creation rate also becomes independent of the electromagnetic fields. Our chief aim in this paper was to investigate the role of the magnetic field strength on the Bell violation. We have seen that, subject to the choices of the initial states, the behavior of the Bell violation can be qualitatively different, e.g., Figs. 2 and 3. For the case of the $\alpha$ vacua, on the other hand, we have also taken into account the variation of the parameter $\alpha$, e.g., Fig. 5.
The above analysis can be attempted to be extended in a few interesting scenarios. For example, instead of having only constant electromagnetic fields, can we also have fluctuating ones, like electromagnetic radiation? Can one also include the effect of gravitational radiation? Finally, it seems also interesting to perform similar analysis in the Rindler spacetime, for its relevance to the near horizon geometry of nonextremal black holes. Discussion of the Schwinger pair creation for a complex scalar field coupled to a constant background electric field in the Rindler spacetime can be seen in [67]. Finally, as we have discussed in Sec. I, it will be important to compute the breaking of scale invariance of the cosmological power spectra in the presence of primordial electromagnetic fields and also to compute the Bell violation by the photons (interacting with the entangled fermions) coming from very distant sources, with the hope to constrain the strengths of those background fields. A gauge invariant formulation of an effective action for the second problem seems to be a nontrivial task. We hope to come back to this issue in future works.

\section{ACKNOWLEDGMENTS}

M. S. A. is fully supported by the Institute Scheme for Innovative Research and Development (ISIRD) Grant No. 9-252/2016/IITRPR/708. S. B. is partially supported by the ISIRD Grant No. 9-289/2017/IITRPR/704. S. C. is partially supported by the ISIRD Grant No. 9-252/2016/ IITRPR/708.

\section{APPENDIX: EXPLICIT FORM OF THE MODE FUNCTIONS AND NORMALIZATIONS}

The four orthonormal simultaneous eigenvectors $\omega_{s}$ of the operators $\left(M \gamma^{0}+L \gamma^{0} \gamma^{3}\right)$ and $\gamma^{1} \gamma^{2}$ appearing in (10) are given by,

$$
\begin{aligned}
& \omega_{1}=\frac{1}{P_{1}}\left(\begin{array}{c}
\frac{\sqrt{M^{2}+L^{2}}-L}{M} \\
0 \\
1 \\
0
\end{array}\right), \quad \omega_{2}=\frac{1}{P_{2}}\left(\begin{array}{c}
0 \\
\frac{\sqrt{M^{2}+L^{2}}+L}{M} \\
0 \\
1
\end{array}\right) \text {, } \\
& \omega_{3}=\frac{1}{P_{1}}\left(\begin{array}{c}
0 \\
\frac{\sqrt{M^{2}+L^{2}}-L}{M} \\
0 \\
-1
\end{array}\right), \quad \omega_{4}=\frac{1}{P_{2}}\left(\begin{array}{c}
\frac{\sqrt{M^{2}+L^{2}}+L}{M} \\
0 \\
-1 \\
0
\end{array}\right) \text {, }
\end{aligned}
$$

where $P_{1}$ and $P_{2}$ are normalization constants. $\omega_{3}$ and $\omega_{4}$ are, respectively, related to $\omega_{1}$ and $\omega_{2}$ via the charge conjugation, $\omega_{3,4}=\mathcal{C} \omega_{1,2}^{*}$, where $\mathcal{C}=i \gamma^{2}$. The explicit representation of the gamma matrices we are using is given by 


$$
\gamma^{0}=\left(\begin{array}{cc}
0 & I \\
I & 0
\end{array}\right), \quad \gamma^{i}=\left(\begin{array}{cc}
0 & \sigma^{i} \\
-\sigma^{i} & 0
\end{array}\right) \quad(i=1,2,3)
$$

We also note here the explicit forms of the positive frequency in and out modes appearing in (20), as follows:

$$
\begin{aligned}
U_{1, n}^{\text {in }}= & \frac{\gamma^{0}}{N_{1} a^{3 / 2}}\left\{\left(i \partial_{\eta}-k_{z} \gamma^{0} \gamma^{3}+a H\left(M \gamma^{0}+L \gamma^{0} \gamma^{3}\right)\right)+\left(i \gamma^{0} \gamma^{2} \partial_{y}-\left(k_{x}+e B y\right) \gamma^{0} \gamma^{1}\right)\right\} \\
& \times e^{-i H L z} e^{i \vec{k} y \cdot \vec{x}} W_{\kappa_{1}, i|\mu|}\left(z_{1}\right) h_{n}(\bar{y}) \omega_{1}, \\
U_{2, n}^{\text {in }}= & \frac{\gamma^{0}}{N_{2} a^{3 / 2}}\left\{\left(i \partial_{\eta}-k_{z} \gamma^{0} \gamma^{3}+a H\left(M \gamma^{0}+L \gamma^{0} \gamma^{3}\right)\right)+\left(i \gamma^{0} \gamma^{2} \partial_{y}-\left(k_{x}+e B y\right) \gamma^{0} \gamma^{1}\right)\right\} \\
& \times e^{-i H L z} e^{i \vec{k} y \cdot \vec{x}} W_{\kappa_{2}, i|\mu|}\left(z_{2}\right) h_{n}(\bar{y}) \omega_{2}, \\
U_{1, n}^{\text {out }}= & \frac{\gamma^{0}}{M_{1} a^{3 / 2}}\left\{\left(i \partial_{\eta}-k_{z} \gamma^{0} \gamma^{3}+a H\left(M \gamma^{0}+L \gamma^{0} \gamma^{3}\right)\right)+\left(i \gamma^{0} \gamma^{2} \partial_{y}-\left(k_{x}+e B y\right) \gamma^{0} \gamma^{1}\right)\right\} \\
& \times e^{-i H L z} e^{i \vec{k} y \cdot \vec{x}} M_{\kappa_{1}, i|\mu|}\left(z_{1}\right) h_{n}(\bar{y}) \omega_{1}, \\
U_{2, n}^{\text {out }}= & \frac{\gamma^{0}}{M_{2} a^{3 / 2}}\left\{\left(i \partial_{\eta}-k_{z} \gamma^{0} \gamma^{3}+a H\left(M \gamma^{0}+L \gamma^{0} \gamma^{3}\right)\right)+\left(i \gamma^{0} \gamma^{2} \partial_{y}-\left(k_{x}+e B y\right) \gamma^{0} \gamma^{1}\right)\right\} \\
& \times e^{-i H L z} e^{i \vec{k} y \cdot \vec{x}} M_{\kappa_{2}, i|\mu|}\left(z_{2}\right) h_{n}(\bar{y}) \omega_{2} .
\end{aligned}
$$

Whereas the negative frequency modes (found via the charge conjugation of the above positive frequency modes, $\left.V \equiv i \gamma^{2} U^{*}\right)$ are given by

$$
\begin{aligned}
V_{1, n}^{\text {in }}= & \frac{\gamma^{0}}{N_{1} a^{3 / 2}}\left\{\left(i \partial_{\eta}-k_{z} \gamma^{0} \gamma^{3}+a H\left(M \gamma^{0}+L \gamma^{0} \gamma^{3}\right)\right)+\left(i \gamma^{0} \gamma^{2} \partial_{y}-\left(k_{x}-e B y\right) \gamma^{0} \gamma^{1}\right)\right\} \\
& \times e^{i H L z} e^{-i \vec{k} y \cdot \vec{x}} W_{-\kappa_{1},-i|\mu|}\left(-z_{1}\right) h_{n}\left(y_{-}\right) \omega_{3}, \\
V_{2, n}^{\text {in }}= & \frac{\gamma^{0}}{N_{2} a^{3 / 2}}\left\{\left(i \partial_{\eta}-k_{z} \gamma^{0} \gamma^{3}+a H\left(M \gamma^{0}+L \gamma^{0} \gamma^{3}\right)\right)+\left(i \gamma^{0} \gamma^{2} \partial_{y}-\left(k_{x}-e B y\right) \gamma^{0} \gamma^{1}\right)\right\} \\
& \times e^{i H L z} e^{-i \vec{k} y \cdot \vec{x}} W_{-\kappa_{2},-i|\mu|}\left(-z_{2}\right) h_{n}\left(y_{-}\right) \omega_{4}, \\
V_{1, n}^{\text {out }}= & \frac{\gamma^{0}}{M_{1} a^{3 / 2}}\left\{\left(i \partial_{\eta}-k_{z} \gamma^{0} \gamma^{3}+a H\left(M \gamma^{0}+L \gamma^{0} \gamma^{3}\right)\right)+\left(i \gamma^{0} \gamma^{2} \partial_{y}-\left(k_{x}-e B y\right) \gamma^{0} \gamma^{1}\right)\right\} \\
& \times e^{i H L z} e^{-i k k_{y} \cdot \vec{x}} M_{-\kappa_{1},-i|\mu|}\left(-z_{1}\right) h_{n}\left(y_{-}\right) \omega_{3}, \\
V_{2, n}^{\text {out }}= & \frac{\gamma^{0}}{M_{2} a^{3 / 2}}\left\{\left(i \partial_{\eta}-k_{z} \gamma^{0} \gamma^{3}+a H\left(M \gamma^{0}+L \gamma^{0} \gamma^{3}\right)\right)+\left(i \gamma^{0} \gamma^{2} \partial_{y}-\left(k_{x}-e B y\right) \gamma^{0} \gamma^{1}\right)\right\} \\
& \times e^{i H L z} e^{-i \vec{k} y \cdot \vec{x}} M_{-k_{2},-i|\mu|}\left(-z_{2}\right) h_{n}\left(y_{-}\right) \omega_{4} .
\end{aligned}
$$

The normalization constants $N_{1}, N_{2}, M_{1}$, and $M_{2}$ are given by (21). We shall explicitly evaluate $N_{1}$ below. The rest can be derived in a similar manner. Using Eqs. (A1) and (A3), we find after some algebra 


$$
\begin{aligned}
\int d^{3} x a^{3} U_{1}^{\dagger \text { in }} U_{1}^{\text {in } \prime}= & \frac{1}{N_{1}^{2}} \int d^{3} x e^{-i\left(\vec{k}_{y} \vec{k}_{y}^{\prime}\right) \cdot \vec{x}} \\
& \times\left[\left(-i \partial_{\eta} W_{\kappa_{1}, i|\mu|}\left(z_{1}\right)^{*}-\left(\frac{k_{z} L}{\sqrt{M^{2}+L^{2}}}-a H \sqrt{M^{2}+L^{2}}\right) W_{\kappa_{1}, i|\mu|}^{*}\left(z_{1}\right)\right) h_{n}(\bar{y}) \omega_{1}^{\dagger}\right. \\
& \left.+\frac{k_{z} M}{\sqrt{M^{2}+L^{2}}} X_{1}^{\dagger} W_{\kappa_{1}, i|\mu|}^{*}\left(z_{1}\right) h_{n}(\bar{y})-\left(\partial_{y} h_{n}(\bar{y})+\left(k_{x}+e B y\right) h_{n}(\bar{y})\right) W_{\kappa_{1}, i|\mu|}^{*}\left(z_{1}\right) \omega_{3}^{\dagger}\right] \\
& \times\left[\left(-i \partial_{\eta} W_{\kappa_{1}, i|\mu|}\left(z_{1}\right)-\left(\frac{k_{z} L}{\sqrt{M^{2}+L^{2}}}-a H \sqrt{M^{2}+L^{2}}\right) W_{\kappa_{1}, i|\mu|}\left(z_{1}\right)\right) h_{n}(\bar{y}) \omega_{1}\right. \\
& \left.+\frac{k_{z} M}{\sqrt{M^{2}+L^{2}}} X_{1} W_{\kappa_{1}, i|\mu|}\left(z_{1}\right) h_{n}(\bar{y})-\left(\partial_{y} h_{n}(\bar{y})+\left(k_{x}+e B y\right) h_{n}(\bar{y})\right) W_{\kappa_{1}, i|\mu|}\left(z_{1}\right) \omega_{3}\right] .
\end{aligned}
$$

The $x$ and $z$ integrals trivially give $\delta\left(k_{x}-k_{x}^{\prime}\right) \delta\left(k_{z}-k_{z}^{\prime}\right) \equiv \delta^{2}\left(\vec{k}_{y}-\vec{k}_{y}^{\prime}\right)$. Using the orthonormality of $\omega_{1}$ and $\omega_{3}$, and the definition of the variable $\bar{y}$ appearing below (14), the $y$ integral is extracted to be

$$
\int d \bar{y}\left[\partial_{y} h_{n}(\bar{y}) \partial_{y} h_{n^{\prime}}(\bar{y})+\bar{y} h_{n}(\bar{y}) \partial_{y} h_{n^{\prime}}(\bar{y})+\bar{y} h_{n^{\prime}}(\bar{y}) \partial_{y} h_{n}(\bar{y})+\bar{y}^{2} h_{n^{\prime}}(\bar{y}) h_{n}(\bar{y})\right] .
$$

Using some properties of the Hermite polynomials [56], the first term equals

$$
\int d \bar{y} \partial_{y} h_{n}(\bar{y}) \partial_{y} h_{n^{\prime}}(\bar{y})=3 e B\left(n+\frac{1}{6}\right) \delta_{n n^{\prime}}
$$

Second and third integrals vanish,

$$
\int d \bar{y} \bar{y} h_{n}(\bar{y}) \partial_{y} h_{n^{\prime}}(\bar{y})=0=\int d \bar{y} \bar{y} h_{n^{\prime}}(\bar{y}) \partial_{y} h_{n}(\bar{y}),
$$

whereas the fourth integral equals

$$
\int d \bar{y} \partial_{y} h_{n}(\bar{y}) \partial_{y} h_{n^{\prime}}(\bar{y}) y_{+}^{2}=e B\left(n+\frac{1}{2}\right) \delta_{n n^{\prime}} .
$$

Collecting all the pieces, the $\bar{y}$ integral becomes

$$
4 e B\left(n+\frac{1}{4}\right) \delta_{n n^{\prime}}
$$

Since normalization is time independent, we may choose the arguments of the Whittaker functions in (A11) as per our convenience. Accordingly, we choose $\eta \rightarrow-\infty$, for which $W_{\kappa_{1}, i|\mu|} \approx e^{-z_{1} / 2} z_{1}^{\kappa_{1}}$. We have

$$
\begin{aligned}
& W_{\kappa_{1}, i|\mu|}\left(z_{1}\right)\left(W_{\kappa_{1}, i|\mu|}\left(z_{1}\right)\right)^{*}=e^{\pi\left|\kappa_{1}\right| \operatorname{sgn}\left(k_{z}\right)}, \quad \partial_{\eta} W_{\kappa_{1}, i|\mu|}\left(z_{1}\right) \partial_{\eta}\left(W_{\kappa_{1}, i|\mu|}\left(z_{1}\right)\right)^{*}=e^{\pi\left|\kappa_{1}\right| \operatorname{sgn}\left(k_{z}\right)}\left(k_{z}^{2}+S_{1}\right), \\
& \partial_{\eta}\left(W_{\kappa_{1}, i|\mu|}\left(z_{1}\right)\right)^{*} W_{\kappa_{1}, i|\mu|}\left(z_{1}\right)-\partial_{\eta} W_{\kappa_{1}, i|\mu|}\left(z_{1}\right)\left(W_{\kappa_{1}, i|\mu|}\left(z_{1}\right)\right)^{*}=2 i e^{\pi\left|\kappa_{1}\right| \operatorname{sgn}\left(k_{z}\right)} \sqrt{k_{z}^{2}+S_{1}} .
\end{aligned}
$$

Putting everything together in (A11), we find the normalization integral becomes $\delta^{2}\left(\vec{k}_{y}-\vec{k}_{y}^{\prime}\right) \delta_{n n^{\prime}}$, with the choice

$$
N_{1}=e^{\pi\left|\kappa_{1}\right| \operatorname{sgn}\left(k_{z}\right) / 2}
$$

The normalization for the other in modes can be found in a similar manner.
For the normalization of the out modes, we choose the integration hypersurface to be in the asymptotic future $\eta \rightarrow 0^{-}$for our convenience and use in this limit

$$
M_{\kappa_{1}, i|\mu|} \approx\left(2 i \eta \sqrt{k_{z}^{2}+S_{1}}\right)^{1 / 2+i|\mu|}
$$

The rest of the calculations remain the same. 
[1] A. Einstein, B. Podolsky, and N. Rosen, Can quantummechanical description of physical reality be considered complete, Phys. Rev. 47, 777 (1935).

[2] S. Bell, On the Einstein-Podolsky-Rosen paradox, Physics 1, 195 (1964).

[3] J. F. Clauser, M. A. Horne, A. Shimony, and R. A. Holt, Proposed Experiment to Test Local Hidden-Variable Theories, Phys. Rev. Lett. 23, 880 (1969).

[4] R. F. Werner, Quantum states with Einstein-Podolsky-Rosen correlations admitting a hidden-variable model, Phys. Rev. A 40, 4277 (1989).

[5] G. Vidal, Entanglement monotones, J. Mod. Opt. 47, 355 (2000).

[6] R. Horodecki, P. Horodecki, and M. Horodecki, Violating Bell inequality by mixed spin- $\frac{1}{2}$ states: Necessary and sufficient condition, Phys. Lett. A 200, 340 (1995).

[7] M. Horodecki, P. Horodecki, and R. Horodecki, Separability of mixed states: Necessary and sufficient conditions, Phys. Lett. A 223, 1 (1996).

[8] S. Yu, Z. Chen, J. Pan, and Y. D. Zhang, Classifying $N$-qubit Entanglement via Bell's Inequalities, Phys. Rev. Lett. 90, 080401 (2003).

[9] P. Y. Chang, S. K. Chu, and C. P. Ma, Bell's inequality and entanglement in qubits, J. High Energy Phys. 09 (2017) 100.

[10] A. Aspect, P. Grangier, and G. Roger, Experimental Tests of Realistic Local Theories via Bell's Theorem, Phys. Rev. Lett. 47, 460 (1981).

[11] A. Aspect, J. Dalibard, and G. Roger, Experimental Test of Bell's Inequalities Using Time Varying Analyzers, Phys. Rev. Lett. 49, 1804 (1982).

[12] N. Gisin and H. B. Pasquinucci, Bell inequality, Bell states and maximally entangled states for $n$ qubits, Phys. Lett. A 246, 1 (1998).

[13] N. D. Mermin, Extreme Quantum Entanglement in a Superposition of Macroscopically Distinct States, Phys. Rev. Lett. 65, 1838 (1990).

[14] A. V. Belinski and D. N. Klyshko, Interference of light and Bell's theorem, Phys. Usp. 36, 653 (1993).

[15] M. B. Plenio and S. Virmani, An introduction to entanglement measures, Quantum Inf. Comput. 7, 1 (2007).

[16] M. A. Nielsen and I. L. Chuang, Quantum Computation and Information Theory (Cambridge University Press, Cambridge, England, 2010).

[17] H. S. Dhar, A. K. Pal, D. Rakshit, A. S. De, and U. Sen, Monogamy of quantum correlations - a review, arXiv: $1610.01069 \mathrm{v} 1$.

[18] B. Richter, K. Lorek, A. Dragan, and Y. Omar, Effect of acceleration on localized fermionic Gaussian states: From vacuum entanglement to maximally entangled states, Phys. Rev. D 95, 076004 (2017).

[19] I. Fuentes-Schuller and R. B. Mann, Alice Falls into a Black Hole: Entanglement in Noninertial Frames, Phys. Rev. Lett. 95, 120404 (2005).

[20] P. M. Alsing, I. F. Schuller, R. B. Mann, and T. E. Tessier, Entanglement of Dirac fields in non-inertial frames, Phys. Rev. A 74, 032326 (2006).

[21] N. Friis, P. Khler, E. Martin-Martinez, and R. A. Bertlmann, Residual entanglement of accelerated fermions is not nonlocal, Phys. Rev. A 84, 062111 (2011).
[22] B. Richter and Y. Omar, Degradation of entanglement between two accelerated parties: Bell states under the Unruh effect, Phys. Rev. A 92, 022334 (2015).

[23] J. Maldacena and G. L. Pimentel, Entanglement entropy in de Sitter space, J. High Energy Phys. 02 (2013) 038.

[24] J. Maldacena, A model with cosmological Bell inequalities, Fortschr. Phys. 64, 10 (2016).

[25] S. Kanno and J. Soda, Infinite violation of Bell inequalities in inflation, Phys. Rev. D 96, 083501 (2017).

[26] I. Fuentes, R. B. Mann, E. Martin-Martinez, and S. Moradi, Entanglement of Dirac fields in an expanding spacetime, Phys. Rev. D 82, 045030 (2010).

[27] S. Kanno, M. Sasaki, and T. Tanaka, Vacuum state of the dirac field in de Sitter space and entanglement entropy, J. High Energy Phys. 03 (2017) 068.

[28] S. Bhattacharya, S. Chakrabortty, and S. Goyal, Emergent $\alpha$-like fermionic vacuum structure and entanglement in the hyperbolic de Sitter spacetime, Eur. Phys. J. C 79, 799 (2019).

[29] S. Bhattacharya, S. Chakrabortty, and S. Goyal, Dirac fermion, cosmological event horizons and quantum entanglement, Phys. Rev. D 101, 085016 (2020).

[30] S. Bhattacharya, H. Gaur, and N. Joshi, Some measures for fermionic entanglement in the cosmological de Sitter spacetime, Phys. Rev. D 102, 045017 (2020).

[31] S. Choudhury, S. Panda, and R. Singh, Bell violation in the Sky, Eur. Phys. J. C 77, 60 (2017).

[32] S. Choudhury and S. Panda, Entangled de Sitter from stringy axionic Bell pair I: An analysis using Bunch-Davies vacuum, Eur. Phys. J. C 78, 52 (2018).

[33] L.E. Parker and D. J. Toms, Quantum Field Theory in Curved Spacetime: Quantized Field and Gravity (Cambridge University Press, Cambridge, England, 2009).

[34] Z. Ebadi and B. Mirza, Entanglement generation by electric field background, Ann. Phys. (Amsterdam) 351, 363 (2014).

[35] Y. Li, Y. Dai, and Y. Shi, Pairwise mode entanglement in Schwinger production of particle-antiparticle pairs in an electric field, Phys. Rev. D 95, 036006 (2017).

[36] A. Agarwal, D. Karabali, and V. Nair, Gauge-invariant variables and entanglement entropy, Phys. Rev. D 96, 125008 (2017).

[37] Y. Li, Q. Mao, and Y. Shi, Schwinger effect of a relativistic boson entangled with a qubit, Phys. Rev. A 99, 032340 (2019).

[38] D. Karabali, S. Kurkcuoglu, and V. Nair, Magnetic field and curvature effects on pair production II: Vectors and implications for chromodynamics, Phys. Rev. D 100, 065006 (2019).

[39] D. C. Dai, State of a particle pair produced by the Schwinger effect is not necessarily a maximally entangled Bell state, Phys. Rev. D 100, 045015 (2019).

[40] S. Bhattacharya, S. Chakrabortty, H. Hoshino, and S. Kaushal, Background magnetic field and quantum correlations in the Schwinger effect, Phys. Lett. B 811, 135875 (2020).

[41] V. Balasubramanian, M. B. McDermott, and M. V. Raamsdonk, Momentum-space entanglement and renormalization in quantum field theory, Phys. Rev. D 86, 045014 (2012). 
[42] G. Grignani and G. W. Semenoff, Scattering and momentum space entanglement, Phys. Lett. B 772, 699 (2017).

[43] S. Ryu and T. Takayanagi, Holographic Derivation of Entanglement Entropy from AdS/CFT, Phys. Rev. Lett. 96, 181602 (2006).

[44] S. Ryu and T. Takayanagi, Aspects of holographic entanglement entropy, J. High Energy Phys. 08 (2006) 045.

[45] D. Boyanovsky, Imprint of entanglement entropy in the power spectrum of inflationary fluctuations, Phys. Rev. D 98, 023515 (2018).

[46] D. Rauch, J. Handsteiner, A. Hochrainer, J. Gallicchio, A. S. Friedman, C. Leung, B. Liu, L. Bulla, S. Ecker, F. Steinlechner et al., Cosmic Bell Test Using Random Measurement Settings from High-Redshift Quasars, Phys. Rev. Lett. 121, 080403 (2018).

[47] M. J. P. Morse, Statistical bounds on CMB Bell violation, arXiv:2003.13562.

[48] K. Subramanian, The origin, evolution and signatures of primordial magnetic fields, Rep. Prog. Phys. 79, 076901 (2016).

[49] M. B. Fröb, J. Garriga, S. Kanno, M. Sasaki, J. Soda, T. Tanaka, and A. Vilenkin, Schwinger effect in de Sitter space, J. Cosmol. Astropart. Phys. 04 (2014) 009.

[50] T. Kobayashi and N. Afshordi, Schwinger effect in 4D de Sitter space and constraints on magnetogenesis in the early Universe, J. High Energy Phys. 10 (2014) 166.

[51] C. Stahl, E. Strobel, and S. S. Xue, Fermionic current and Schwinger effect in de Sitter spacetime, Phys. Rev. D 93, 025004 (2016).

[52] E. Bavarsad, C. Stahl, and S. S. Xue, Scalar current of created pairs by Schwinger mechanism in de Sitter spacetime, Phys. Rev. D 94, 104011 (2016).

[53] T. Hayashinaka, T. Fujita, and J. Yokoyama, Fermionic Schwinger effect and induced current in de Sitter space, J. Cosmol. Astropart. Phys. 07 (2016) 101.
[54] E. Bavarsad, S. P. Kim, C. Stahl, and S. S. Xue, Effect of a magnetic field on Schwinger mechanism in de Sitter spacetime, Phys. Rev. D 97, 025017 (2018).

[55] A. Mironov, A. Morozov, and T. N. Tomaras, Geodesic deviation and particle creation in curved spacetimes, Pis'ma Zh. Eksp. Teor. Fiz. 94, 872 (2011).

[56] M. Abramowitz and I. Stegun, Handbook of Mathematical Functions with Formulas, Graphs, and Mathematical Tables (National Bureau of Standards, Washington, DC, 1964).

[57] B. Allen, Vacuum states in de Sitter space, Phys. Rev. D 32, 3136 (1985).

[58] J. de Boer, V. Jejjala, and D. Minic, Alpha-states in de Sitter space, Phys. Rev. D 71, 044013 (2005).

[59] E. Mottola, Particle creation in de Sitter space, Phys. Rev. D 31, 754 (1985).

[60] M. B. Einhorn and F. Larsen, Interacting quantum field theory in de Sitter vacua, Phys. Rev. D 67, 024001 (2003).

[61] A. Bedroya, de Sitter complementarity, TCC, and the swampland, arXiv:2010.09760.

[62] H. Collins, Fermionic alpha-vacua, Phys. Rev. D 71, 024002 (2005).

[63] N. Iizuka, T. Noumi, and N. Ogawa, Entanglement entropy of de Sitter space $\alpha$-vacua, Nucl. Phys. B910, 23 (2016).

[64] S. Kanno, J. Murugan, J. P. Shock, and J. Soda, Entanglement entropy of $\alpha$-vacua in de Sitter space, J. High Energy Phys. 07 (2014) 072.

[65] Y. Kwon, No survival of nonlocalilty of fermionic quantum states with alpha vacuum in the infinite acceleration limit, Phys. Lett. B 748, 204 (2015).

[66] S. Choudhury and S. Panda, Quantum entanglement in de Sitter space from stringy axion: An analysis using $\alpha$ vacua, Nucl. Phys. B943, 114606 (2019).

[67] C. Gabriel and P. Spindel, Quantum charged fields in Rindler space, Ann. Phys. (Amsterdam) 284, 263 (2000). 